\title{
Compressed Natural Gas Vehicle Maintenance Facility Modification Handbook
}

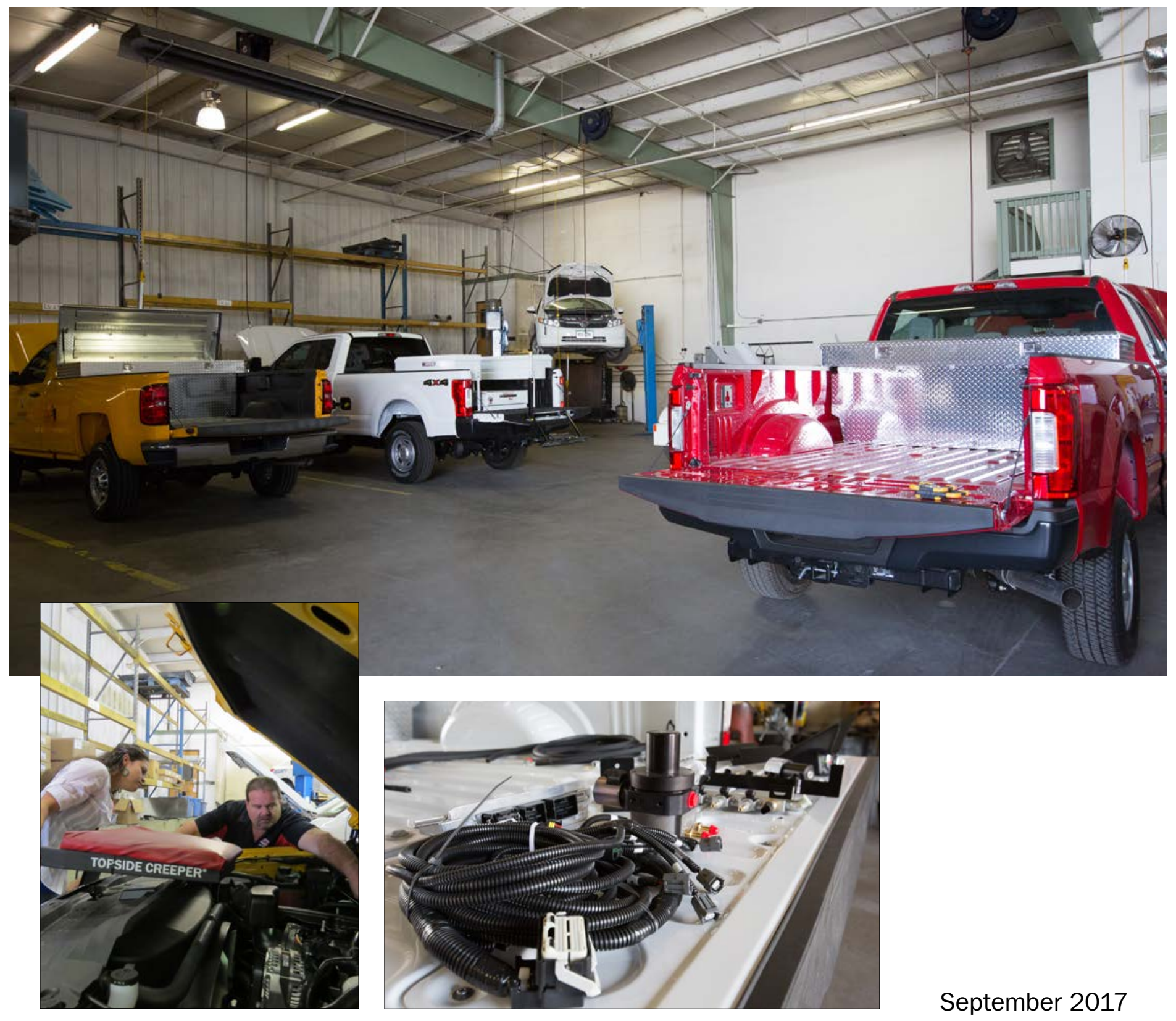




\section{Acknowledgments}

This guide provides a review of all aspects of indoor compressed natural gas vehicle maintenance facility protection, including the initial assessment of the facility, code compliance requirements, and specific design criteria. The authors would like to acknowledge Gladstein, Neandross \& Associates in providing considerable information and photos during the development of this handbook.

\section{Authors}

Kay Kelly and Margo Melendez, National Renewable Energy Laboratory

\section{Co-Authors}

John Gonzales and Lauren Lynch, National Renewable Energy Laboratory Bob Coale and Jarrod Kohout, Gladstein, Neandross \& Associates

\section{Work Sponsor}

The U.S. Department of Energy Vehicle Technologies Office, Clean Cities program, and Dennis A. Smith and Mark S. Smith

\section{Reviewers}

Steve Saltzgiver, Mercury Associates, Inc. Ted Barnes, Gas Technology Institute

Rob Adams, Marathon Technical Services, LLC

Andrew Klock and Susan Bershad, National Fire Protection Association

Stephe Yborra, Yborra \& Associates, LLC

\section{Disclaimer}

This report was prepared as an account of work sponsored by an agency of the United States government. Neither the United States government nor any agency thereof, nor any of their employees, makes any warranty, express or implied, or assumes any legal liability or responsibility for the accuracy, completeness, or usefulness of any information, apparatus, product, or process disclosed, or represents that its use would not infringe privately owned rights. Reference herein to any specific commercial product, process, or service by trade name, trademark, manufacturer, or otherwise does not necessarily constitute or imply its endorsement, recommendation, or favoring by the United States government or any agency thereof. The views and opinions of authors expressed herein do not necessarily state or reflect those of the United States government or any agency thereof.

Every effort has been made to ensure that this manual is accurate, complete, and comprehensive at the time of publication. It is intended to be used as a guide and resource document. The authors strongly encourage all parties with an interest in establishing compressed natural gas vehicle maintenance facilities to engage professional support to ensure code compliance and optimal systems operation.

This document is not intended for use as a "how to" guide for individuals or organizations performing facility modifications. 


\section{Glossary}

Air Changes per Hour (ACH): A measurement of the number of volumetric air exchanges within a structure caused by natural or mechanical ventilation.

Authority Having Jurisdiction (AHJ): Person(s) or agency responsible for reviewing, approving, and permitting proposed modifications, and issuing construction and occupancy permits. For the purposes of this document an AHJ is an agency.

Class I, Division 1 Electrical Hazard Zone: A zone that is expected to contain hazardous concentrations of gas or vapor in the event of a spill or leak which, therefore, must be provided with ignition source protection.

Class I, Division 2 Electrical Hazard Zone: A zone which may contain hazardous concentrations of gas or vapor and, therefore, must be protected. For compressed natural gas vehicle maintenance facilities, Class I, Division 2 represents conditions in which methane releases could occur but are not expected.

Compressed Natural Gas (CNG): Natural gas that consists primarily of methane and small amounts of ethane, propane, and heavier hydrocarbons and has been compressed and stored in high-pressure vessels at pressures up to 5,000 pounds per square inch (psi).

Creditable Release: A release of natural gas within a repair garage that results in a plume of sufficient concentration that it exceeds the lower flammability limit.

Design-Build (DB): A type of contract whereby the selected contractor is responsible for the complete design of the facility, securing permits, procurement, construction, commissioning, startup, and often maintenance activities.

Design-Bid-Build (DBB): A type of contract whereby one consultant is responsible for preparing the facility design and permitting documents while a building contractor is responsible for pulling permits, procuring equipment and materials, construction, commissioning, startup, and sometimes maintenance activities.

Hot Work: A type of maintenance work involving the use of oxyacetylene torches and/or electric arc welders. This could also include the use of grinders or other sparkproducing tools.

Ignition Source: A spark, flame, electricity, static electricity, or hot surface capable of igniting a fuel that is within its combustible range.
International Fire Code (IFC): A guidance document commonly used with, or in lieu of, NFPA 30A that covers the design of vehicle maintenance facilities and protection systems.

International Mechanical Code (IMC): A guidance document that establishes regulations for the design and operation of mechanical systems based on prescriptive and performance-based provisions commonly used with, or in lieu of, NFPA 30A.

Lower Flammability Limit (LFL): The minimum concentration of a combustible gas that will support combustion; sometimes referred to as the lower explosive limit (LEL). This is generally accepted to be $5 \%$ natural gas in air by volume at atmospheric conditions.

Major Repair Garage: A maintenance facility in which major repairs are performed, such as engine overhauls, vehicle chassis and body repairs, and similar work, that require emptying the vehicle's fuel tank. Any work that involves service to a vehicle's fuel system also falls into this category.

Make-up Air: Fresh air that replaces air that is exhausted from a structure.

Methane $\left(\mathrm{CH}_{4}\right)$ : The primary component of natural gas.

Minor Repair Garage: A maintenance facility in which minor repairs are performed, such as lubrication, engine tune-ups, replacement of parts and tires, fluid changes, and similar maintenance work, that does not require emptying a vehicle's fuel tank. Any work that involves service to a vehicle's fuel system is not permitted in a Minor Repair Garage.

National Fire Protection Association (NFPA): NFPA is a global, nonprofit organization devoted to eliminating death, injury, and property and economic loss due to fire, electrical hazards, and related threats. NFPA's codes and standards are designed to minimize the risk and effects of fire by establishing criteria for building, processing, design, service, and installation in the United States, as well as many other countries. NFPA 30A is the basic document used in conjunction with maintenance facilities. Portions of NFPA 88A may also apply.

Natural Gas: Natural gas used as a vehicle fuel is composed of $90 \%-95 \%$ methane and other longer-chain simple hydrocarbons. When used in the gaseous state, natural gas 
is compressed to pressures up to 5,000 psi and is commonly known as compressed natural gas (CNG). Natural gas is odorized with mercaptan to yield the familiar "rotten egg" smell.

Natural Gas Vehicle: A motor vehicle with a combustion engine fueled by natural gas.

Paths of Migration: The flow paths by which natural gas travels as it dissipates throughout a structure.

Pressure Relief Device (PRD): A valve activated by high temperature or pressure used to eliminate overpressure in a vehicle fuel tank or system. A temperature-activated valve is designed to open at a given temperature (for example in the event of a fire) and remain open. Once the device is activated, the valve remains permanently open and cannot be reset to the closed position. A pressure-activated valve is designed to open at a given pressure and will remain open until the overpressure is released. This type of valve is resettable and will reseal when the lower prescribed pressure is realized.

Pressure Release Valve (PRV): A type of valve used to control or limit the pressure in a system or vessel. A PRV is usually resettable and when activated by high pressure will open to release the overpressure until a lower prescribed pressure is realized, then reseal. PRVs are usually found on stationary tanks and equipment and can also be used for vehicle pressure regulators. PRVs are also known as safety relief valves (SRVs).

Upper Flammability Limit (UFL): The maximum concentration of a combustible gas that will ignite; sometimes referred to as the upper explosive limit (UEL). This is generally accepted to be $15 \%$ natural gas in air by volume at atmospheric conditions.
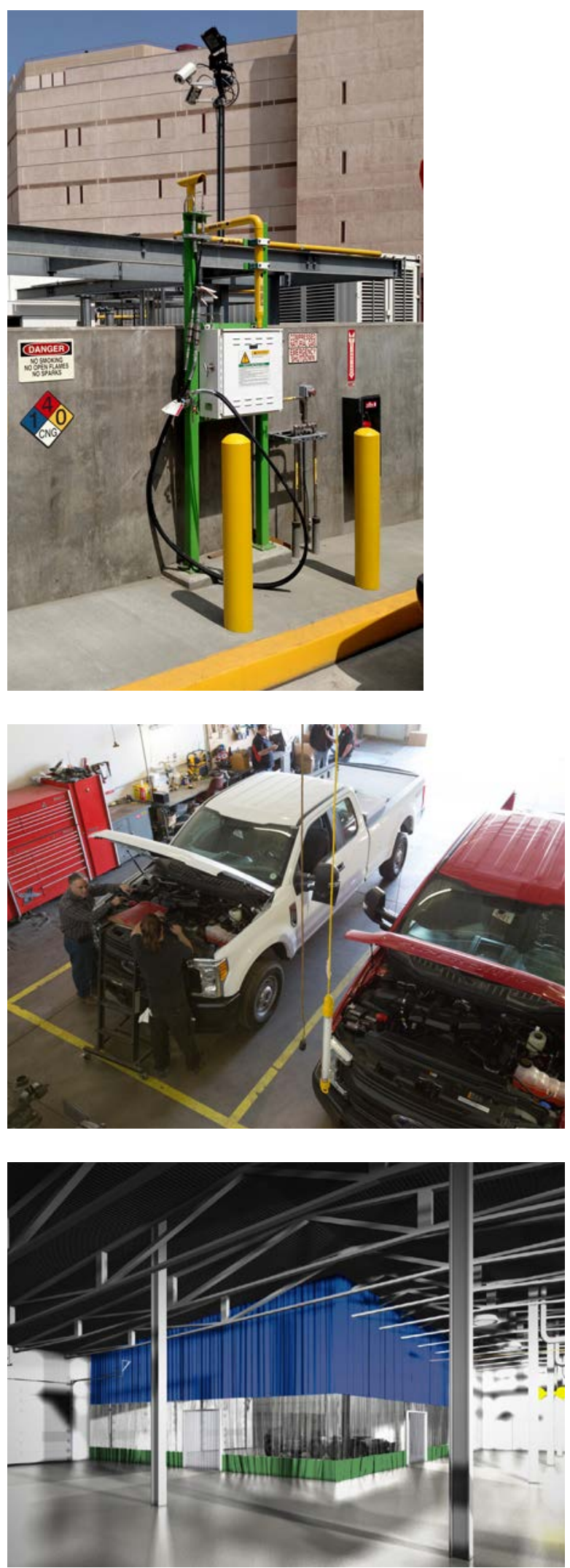


\section{Contents}

Introduction $\ldots \ldots \ldots \ldots \ldots \ldots \ldots \ldots \ldots \ldots \ldots \ldots \ldots \ldots \ldots \ldots \ldots \ldots \ldots$

Regulations $\ldots \ldots \ldots \ldots \ldots \ldots \ldots \ldots \ldots \ldots \ldots \ldots \ldots \ldots \ldots \ldots \ldots \ldots \ldots \ldots \ldots \ldots$

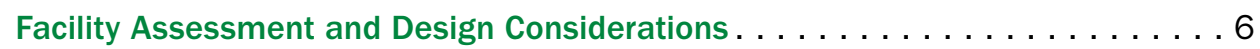

Paths of Migration . . . . . . . . . . . . . . . . 8

Ventilation . . . . . . . . . . . . . . . . . . . . . 10

Space Heating . . . . . . . . . . . . . . . . . . . . . . . . . . . . . 15

Electrical Wiring, Lighting, and Appliance Considerations . . . . . . . . 16

Gas Detection, Alarm, and Control Systems . . . . . . . . . . . . . 19

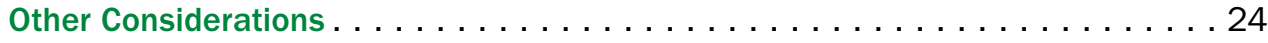

Vehicle Fuel System Defueling. . . . . . . . . . . . . . . . . 24

Engine Exhaust Extractors. . . . . . . . . . . . . . . . . . . . 24

Isolation of Non-Work Areas . . . . . . . . . . . . . . . 25

Automatic Shut-Off of Building Gas Supply . . . . . . . . . . . . . 25

Associated Bays. . . . . . . . . . . . . . . . . . . . . 26

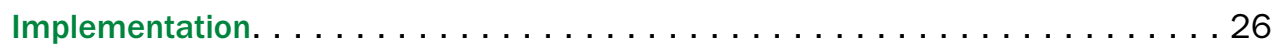

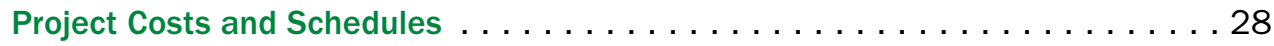

Facility Modification Costs. . . . . . . . . . . . . . . . . 28

Facility Modification Timelines . . . . . . . . . . . . . . . . . 30

Appendix A. Specific Properties of Common Vehicle Fuels . . . . . . . . . 31

Appendix B. Preliminary Maintenance Facility Assessment . . . . . . . . . . 32

Appendix C. Minor/Major Maintenance Facility Assessment . . . . . . . . . 34 


\section{List of Figures}

Figure 1. Unsealed conduit or pipes passing through walls or ceilings present a potential path of migration...9

Figure 2. Structural members passing through a wall present a potential path of migration .........................10

Figure 3. Gaps between walls and the corrugated ceiling panels present a potential path of migration..........10

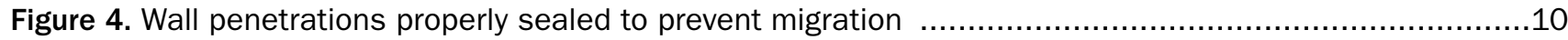

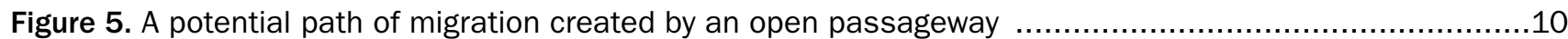

Figure 6. Examples of stairways to upper levels that are potential paths of migration .............................10

Figure 7. A pass-through window presents a potential path of migration .............................................11

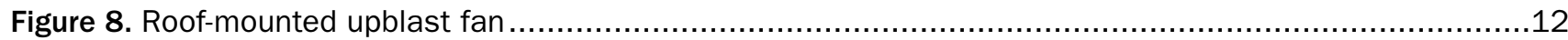

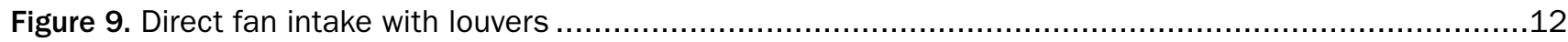

Figure 10. Intake ducting used to evacuate air from specific areas ...................................................12

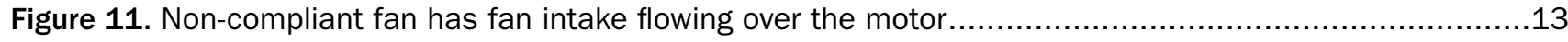

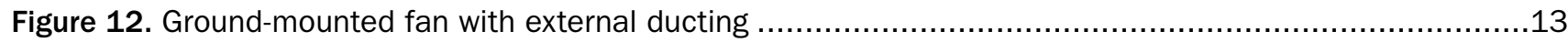

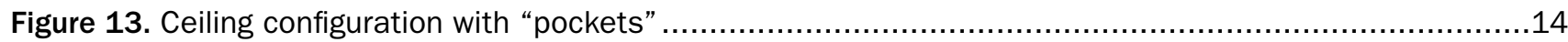

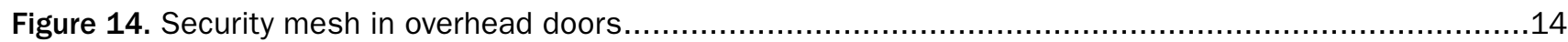

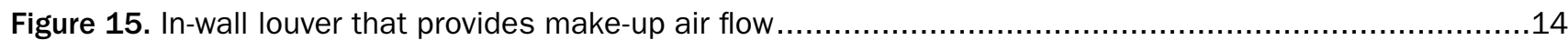

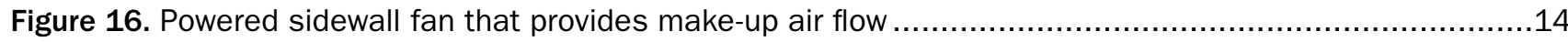

Figure 17. Conventional fuel maintenance facility ventilation pickup points .........................................15

Figure 18. Gas-fired infrared tube-type heater needs to be replaced or removed .....................................16

Figure 19. Electric infrared radiant heater needs to be replaced or removed ..........................................16

Figure 20. Floor-mounted portable propane heater should be eliminated .................................................16

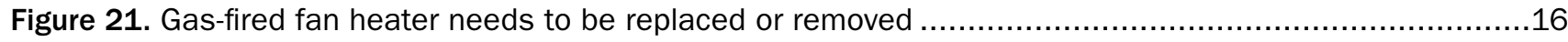

Figure 22. Relocating multiple conduits, such as those shown here, would be costly and complex...............17

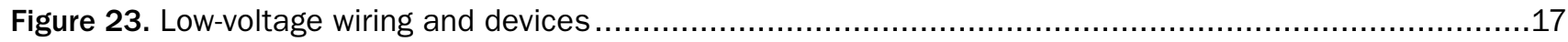

Figure 24. Overhead lighting fixture and ballast that must be relocated .................................................18

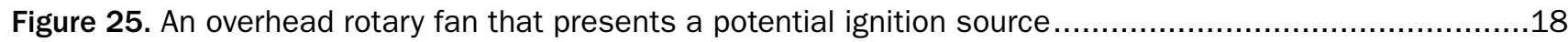

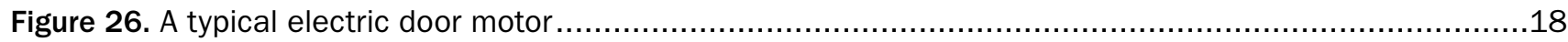

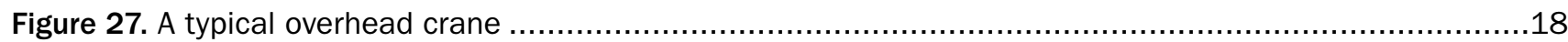

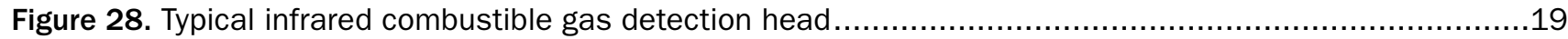

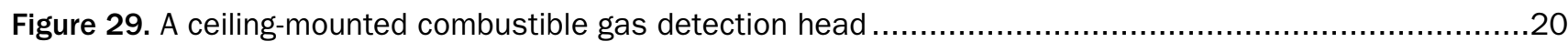

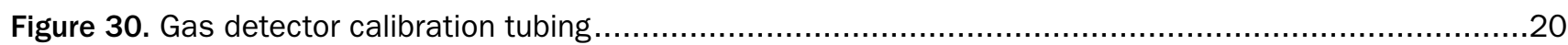

Figure 31. Examples of alarm status lights and horn panels (external and internal)...............................20

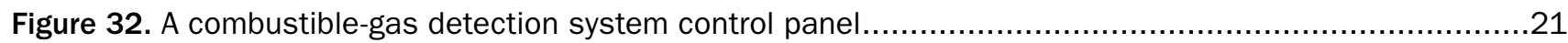

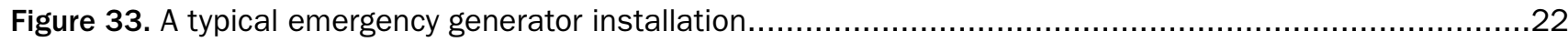

Figure 34. Example of a preliminary facility layout presenting proposed equipment.................................23

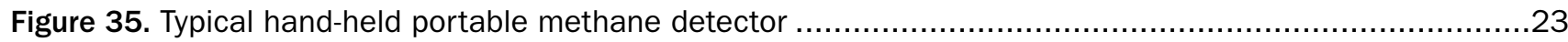

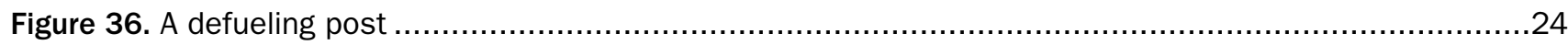

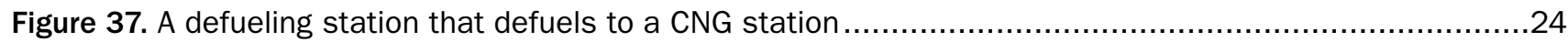

Figure 38. The exhaust from a natural gas engine is hotter than that from a diesel engine, so some exhaust extractor hoses may need to be replaced during the facility modifications. ....................25

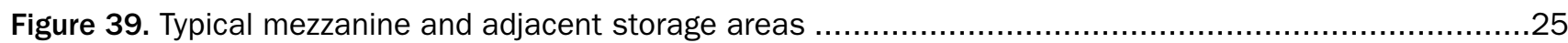

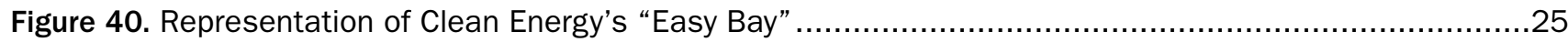

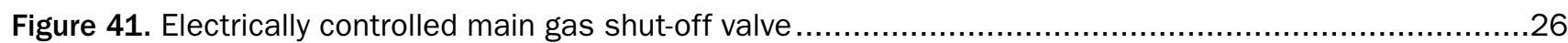

Figure B1. Critical decision path of a CNG vehicle maintenance facility modification ................................33 


\section{List of Tables}

Table 1. Possible Series of Actions Based on the Two Suggested Levels of Detection ............................22

Table 2. CNG Vehicle Maintenance Facility Modification Schedule ....................................................31

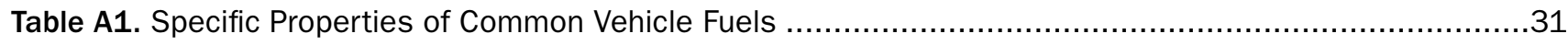




\section{Introduction}

To ensure the safety of personnel and facilities, vehicle maintenance facilities are required by law and by guidelines of the National Fire Protection Association (NFPA), the International Mechanical Code (IMC), and the International Fire Code (IFC) to incorporate certain building/structural design features. They are also required to be fitted with certain fire protection equipment and devices because of the potential for fire or explosion if fuel leaks or spills. All fuels have an explosion or fire potential if specific conditions are present; however, the means of ensuring safety are different based upon the properties of different fuels.

The hazard presented by liquid fuels, such as gasoline and diesel, results from the spillage of these liquids and subsequent ignition of vapors, causing a fire or explosion. Facilities that maintain liquid-fueled vehicles and implement appropriate safety measures are protected with ventilation systems designed to capture liquid fuel vapors at or near floor level. To minimize the potential for ignition in the event of a spill, receptacles, electrical fixtures, and hot-work operations, such as welding, are located outside of these areas.

Facilities that maintain vehicles fueled by natural gas require implementation of different safety measures. Compressed natural gas $(\mathrm{CNG})$ is composed of mostly methane $\left(\mathrm{CH}_{4}\right)$ with slight amounts of other hydrocarbons. $\mathrm{CNG}$ is lighter than air and will therefore rise to the ceiling of the maintenance facility and quickly dissipate rather than remaining at or near floor level like liquid fuel vapors in the event of a release. If concentrations of $5 \%-15 \%$ by volume of natural gas encounter an ignition source, the natural gas may ignite, with potentially serious results. However, due to natural gas's ability to rapidly dissipate, this concentration is rarely seen in practice. The lower flammability limit (LFL) for CNG (5.3\%) is considerably higher than that for gasoline $(1.4 \%)$ or for diesel $(0.6 \%)$. The specific properties of methane, as well as other vehicle fuels, are given in Appendix A.

Facilities that maintain $\mathrm{CNG}$ vehicles indoors must also be protected against fire and explosion. Although some of the means of protection for CNG vehicle maintenance facilities are similar to those used for liquid-fueled vehicles (ventilation and elimination of ignition sources), the types and placement of the protection equipment are different because of the gaseous nature of the fuel and its properties. CNG maintenance facilities may also require additional safeguards, such as combustible-gas detectors and control systems or specialized space heating, which are not needed in facilities servicing liquid-fuel vehicles.

Although some of the means of protection for CNG vehicle maintenance facilities are similar to those used for liquid-fueled vehicles, the types and placement of the equipment are different because of the gaseous nature of the fuel and its properties.

This handbook covers the five primary elements below that must be considered when developing a CNG vehicle maintenance facility design that will protect against the ignition of natural gas releases.

- Ventilation must provide sufficient air flow to reduce the concentration of the released gas and at the same time evacuate the gas from the structure.

- Paths of migration must be controlled to prevent the released gas from entering unprotected areas of the structure.

- Space heating must be designed in accordance with guidelines so that open flames or hot surfaces do not provide an ignition source.

- Electrical wiring and equipment must be installed in such a manner that they do not provide sources of ignition due to sparking. The equipment itself can be designed to be "explosion proof."

- Methane detection and control systems and alarms must provide defense against dangerous concentrations of natural gas by alerting personnel and disabling potential electrical ignition sources.

All are accompanied by the need to establish specific protocols and training to ensure safety. Each of these is discussed in detail in this handbook.

There is inconsistent and conflicting information regarding $\mathrm{CNG}$ vehicle maintenance facility protection requirements within the industry. The purpose of this handbook is to provide a thorough review of all aspects of indoor CNG vehicle maintenance facility protection, including the initial assessment of the facility, code compliance requirements, and specific design criteria. Alternative 
means of providing a safe maintenance environment are also discussed. Research into various safety methods is ongoing, which means that the codes covering facility safety may be updated over time. Always consult with the local authority having jurisdiction (AHJ) to determine the specific codes and editions of codes that will prevail for their specific project.

One of the primary variables in evaluating and modifying a maintenance facility is the intended use of that facility. The codes and regulations offer very different approaches, and it is important to understand that some requirements differ based on the type of maintenance work to be performed within the facility. It should be noted that the local governing body will have the final say as to which code(s) will be followed, and the AHJ will ensure the appropriate codes are followed. Early interaction with the $\mathrm{AHJ}$ is an essential part of the evaluation of facility modifications.

Although similar requirements are needed for the design of new $\mathrm{CNG}$ vehicle maintenance facilities, this handbook has been prepared for use by fleet and maintenance facility managers who are responsible for maintaining CNG vehicles indoors in existing facilities. It is intended to assist managers in evaluating physical modifications or changes in operating protocols, but is not intended to enable them to perform the designs themselves. Rather, it should educate managers about the design variables so that they fully understand the requirements and can ensure compliance. This document is a guide; final designs, equipment selection, and development of operating protocols should be coordinated with a professional engineer(s) registered/licensed in the state where the maintenance facility is located. The engineer(s) is responsible for preparing the drawings that will be used for permitting and construction.

Implementation protocols including design, permitting, and construction alternatives as well as project costs and schedules are also provided in this handbook. A questionnaire and a decision tree (see Appendices B and C, respectively) have been included to aid the user in determining the best approach for the maintenance facility.

This handbook covers maintenance facilities that service CNG-fueled vehicles. Although similar requirements are mandated for liquefied natural gas- or liquefied petroleum gas-fueled vehicles, these are not covered in this handbook.

\section{Receive Technical Assistance from Clean Cities}

The U.S. Department of Energy's (DOE's) Clean Cities program advances the nation's economic, environmental, and energy security by supporting local actions to cut petroleum use in transportation. At the national level, the program provides unbiased and objective, fuelneutral resources and information to help transportation stakeholders evaluate options and achieve goals around alternative fuels, advanced vehicles, and other strategies to promote the use of domestic fuels in transportation. At the local level, nearly 100 Clean Cities coalitions leverage these resources to create networks of local stakeholders that advance transportation projects. These coalitions are public-private partnerships comprising businesses, fuel providers, vehicle fleets, state and local government agencies, and community organizations that share information and resources, inform public policy, educate the public, and collaborate on transportation projects.

Clean Cities coordinators are the primary contacts for their coalitions and work with local fleets. They can assist fleet and facility maintenance managers in locating qualified facility assessors, design engineers, and construction firms with $\mathrm{CNG}$ experience.

Clean Cities coordinators are the primary contacts for their coalitions and can assist fleet and facility maintenance managers in locating qualified facility assessors, design engineers, and construction firms with CNG experience.

Visit cleancities.energy.gov to find a local Clean Cities coordinator.

The Clean Cities program also provides technical assistance and problem-solving through Tiger Teams, a group of highly skilled experts from DOE national laboratories and industry. Tiger Teams can help fleets and industry tackle difficult technical and market challenges. These can include issues related to deploying alternative and renewable fuels, idle-reduction measures, fuel economy improvements, and emerging transportation technologies. For fleets that have already initiated projects, Tiger Teams can help overcome implementation or operational challenges of new technologies, get delayed projects moving again, or serve as a neutral third party to conduct an analysis and identify potential solutions. 
Additionally, they can help address safety or operational issues by identifying the root cause of a problem and determining next steps.

To learn more about which projects are eligible for assistance, visit the Tiger Teams page at cleancities.energy. gov/technical-assistanceltiger-teams/. For more information about Tiger Teams, contact John Gonzales at john. gonzales@nrel.gov, or Kay Kelly atkay.kelly@nrel.gov.

\section{Regulations}

There are a number of codes and ordinances dealing with the design and use of a $\mathrm{CNG}$ vehicle maintenance facility. The primary documents used by AHJs are NFPA 30A - Code for Motor Fuel Dispensing Facilities and Repair Garages, and the appropriate sections of the IFC. Other documents that may be employed include:

- International Building Code

- International Electrical Code

- International Mechanical Code

- NFPA 52 - Vehicular Gaseous Fuel Systems Code

- NFPA 88A - Standard for Parking Structures

- National Electrical Code

- National Fire Code

- National Mechanical Code.

All of these codes provide valid and safe methods for facility design, but it is the local governing body that makes the final decision as to which are used.

These codes generally contain similar (or in many cases identical) language. In addition to those listed above, many local governing bodies have their own additional codes. It is essential to recognize that all of these codes provide valid and safe methods for facility design, but it is the local governing body that makes the final decision as to which are used, and the AHJ that ensures the codes are met.

Approval of the permit applications that contain the designs and proposed equipment for CNG facility modifications is generally the responsibility of local building and safety departments. Typically, the primary responsibility of signing off on a design rests with the local fire department, or another AHJ. Other permitting agencies such as the electrical, mechanical, or structural departments will sign off on construction elements of the design. Compliance inspections may be conducted by all of the agencies.

Local governing bodies adopt specific versions of the above codes, and, until newer versions are officially recognized in a local governing body, the latest editions may not be valid for design purposes. Some local governing bodies may develop requirements in addition to any national or international code they have adopted. For this reason, it is essential that the appropriate approved/ acknowledged/adopted version of the codes and ordinances are used as the basis for design and implementation. Although there is generally agreement between the codes and ordinances prepared by various agencies, in a few cases the requirements may differ.

NFPA 30A provides specific design and operational requirements based on the type of maintenance performed within the facility. This document separates the type of maintenance and repair activities into two categories: major and minor. This distinction is critical in determining the upgrades required to existing facilities.

It is essential that an open dialogue be established with the $\mathrm{AHJ}$ early in the design process so that a mutually acceptable design, including appropriate equipment and operating protocols, is developed.

The codes are intended to be guidelines to help the designer and the AHJ arrive at a practical solution to provide a safe environment within the maintenance facility. The authors of the codes realize there are many varying factors, particularly the overall design of a maintenance facility, its mission, weather conditions, and other situations which preclude a "one-size-fits-all" approach to facility design. It is essential that an open dialogue be established with the AHJ early in the design process so that a mutually acceptable design, including appropriate equipment and operating protocols, is developed.

\section{Facility Assessment and Design Considerations}

The following section provides a practical approach to evaluating an existing maintenance facility for conversion into a facility properly equipped to perform indoor 
maintenance on $\mathrm{CNG}$ vehicles. Facility assessment will involve two steps: first, an evaluation of the fleet to be maintained, and second, a thorough inspection of the existing maintenance facility. The initial part of the evaluation will involve assessing the types of vehicles in the fleet (including potential fleet additions) and the anticipated types of maintenance that will be performed on them. The latter part of the evaluation will include a careful inspection of the existing structure, ventilation, lighting, and heating and electrical systems, all of which may require modification or replacement.

\section{Facility Needs Assessment}

Essential first steps in evaluating the use of an existing facility include an analysis of the nature of the maintenance that will be conducted and the types of vehicles that will be maintained. The types of service conducted within the maintenance facility will dictate the nature and extent of modifications required to comply with the codes and ordinances.

The types of service conducted within the facility will dictate the nature and extent of modifications required to comply with codes and ordinances.

It is important to consider not only the present fleet make-up, but also how the fleet may look in years to come. Usually a CNG fleet grows slowly as conventionally fueled vehicles are replaced; full conversion may take several years. Thus, the maintenance requirements of today may be different in the future.

\section{Classification as a Major or Minor Repair Facility}

NFPA 30A and its counterparts, the IFC and the IMC, are the primary codes that cover vehicle maintenance facilities and the classification of maintenance garages. NFPA 30A provides specific design and operational requirements based on the type of maintenance performed within the facility. This code separates the type of maintenance and repair activities into two categories: major and minor.

A major repair garage is a maintenance facility in which major repairs are performed, such as engine overhauls, vehicle chassis and body repairs, and similar maintenance work that requires emptying the vehicle's fuel tank. Any work that involves service to a vehicle fuel system may only be performed in a major repair garage.
- Major repair garage: A maintenance facility in which major repairs are performed, such as engine overhauls, vehicle chassis and body repairs, and similar maintenance work that requires emptying the vehicle's fuel tank. Any work that involves service to a vehicle fuel system may only be performed in a major repair garage.

- Minor repair garage: A maintenance facility in which minor repairs are performed, such as lubrication, engine tune-ups, replacement of parts and tires, fluid changes, and similar maintenance work, that does not require emptying the vehicle's fuel tank. Any work that involves service to a vehicle's fuel system is not permitted in a minor repair garage.

A minor repair garage is a maintenance facility in which minor repairs are performed, such as lubrication, engine tune-ups, replacement of parts and tires, fluid changes, and similar maintenance work, that does not require emptying the vehicle's fuel tank. Any work that involves service to a vehicle's fuel system is not permitted in a minor repair garage.

It is important to note that it is the facility that is categorized as major or minor, not the vehicles on which maintenance is performed.

The distinctions of a major repair garage versus a minor repair garage are critical because they define the types of protection needed within the facility and the nature of the maintenance work that may be performed within the facility. The protection needed for a minor repair garage is generally less extensive and less costly than that required for a major repair garage because of the nature of the work being performed. Fleet and facility managers should carefully evaluate the makeup of the fleets and the work they expect to be performed to determine which type of garage classification best suits their operations. If ANY work classified as major is to be performed, the facility MUST be classified as a major repair garage, regardless of how infrequent or how small the activity.

\section{Alternative Approaches}

If a fleet is small, consideration could be given to outsourcing vehicle maintenance to a facility suitably authorized for indoor $\mathrm{CNG}$ vehicle repair. For a very small fleet, all maintenance could be performed off site, thereby eliminating the need for any maintenance facility modifications. For these scenarios, a cost-benefit analysis 
that includes possible growth of the $\mathrm{CNG}$ vehicle fleet over time will aid in determining the best option for the fleet. Certain work only allowed in a major repair garage (hot work or engine overhauls, for example) might be performed off site, while minor repair garage work could be conducted on site. This could reduce the complexity and costs of facility modifications.

Another possibility to consider is whether or not major garage maintenance work can be performed outdoors. In some areas, the weather is suitable to allow this either in the open or under a canopy. However, if heavy maintenance is required, outdoor facilities may not have the infrastructure-overhead cranes, pits, or other equipment - necessary to support the work needed, or may not be a suitable environment due to wind, dirt, dust, and abrasive materials that may hamper the maintenance activities.

If outsourcing or off-site maintenance is not cost effective or practical, it is possible that certain areas within the facility can be isolated and modified to perform maintenance on CNG vehicles. This depends on the number of maintenance bays and the layout of the existing facility. In some cases, bays used for the majority of the maintenance activities (minor repairs) can be modified according to the requirements for minor repair garages, and separate, segregated bay(s) can be modified according to the requirements for major repair garages. It is not always necessary to modify an entire maintenance facility to major repair garage standards if such activities can be performed in a separate location that is appropriately protected.

It should be noted that some operational risks may be reduced by establishing operating protocols and training programs. Such protocols may include the appropriate preparation of a $\mathrm{CNG}$ vehicle prior to bringing it into the facility (e.g., checking for fuel system leaks, noting CNG tank pressure and temperature) and ensuring that only maintenance permitted for the garage classification is conducted within the facility.

If there is any doubt regarding the classification of specific work activities, it is recommended that the fleet or facility manager opt for the more stringent codes-those relating to major repair garages.

While every effort can be made to ensure strict adherence to operating protocols, human involvement presents an opportunity, deliberate or not, to deviate from such protocols. Deviation from protocols has the potential to create a dangerous environment as well as violate the codes and ordinances. The fleet or facility manager should critically review any choices being considered that may cause deviations. If there is any doubt regarding the classification of specific work activities, it is recommended that the fleet or facility manager opt for the more stringent codes - those relating to major repair garages.

\section{Identification of Required Upgrades}

Once a maintenance facility has been classified as either a minor or major repair garage, an evaluation of the existing facility should be conducted to determine what modifications will be required to achieve compliance with the respective classification. The following sections of this handbook address the physical elements of the facility that will require evaluation when assessing the modifications necessary to maintain $\mathrm{CNG}$ vehicles:

- Paths of migration

- Ventilation

- Space heating

- Electrical wiring, lighting, and appliance considerations

- Methane detection, alarm, and control systems.

It is essential to use a trained, experienced assessor to conduct the initial inspection of the facility. Whenever possible, up-to-date/as-built drawings of the facility should be provided to the facility assessor. A scaled floor plan of the entire facility is important. Other useful drawings include electrical plans that define the location and capacity of existing switchboards, a listing of existing electrical equipment, and elevation drawings of the facility. Newer facilities may have these drawings available in computer-aided design and drafting format, electronically stored, whereas older facilities may only have paper copies of original construction drawings. In some cases, construction drawings may be unavailable, thereby requiring more time and additional resources for the facility assessor to make an assessment.

\section{Paths of Migration}

Natural gas used as a vehicle fuel is composed mostly of methane $\left(\mathrm{CH}_{4}\right)$ with small amounts of other hydrocarbons. It is lighter than air and will rapidly rise and dissipate once released. When a natural gas release occurs indoors, the gas initially accumulates near the ceiling before dispersing throughout the structure and becoming 
less concentrated. The path by which natural gas travels as it dissipates is referred to as its "path of migration." Because natural gas is normally odorless, the non-toxic chemical mercaptan is added to allow easy detection due to its "rotten egg" smell. Mercaptan provides a powerful first defense in detecting a natural gas leak. The smell can be detected in natural gas concentrations as low as $1 \%$, well below the LFL of $5 \%$.

The path by which natural gas travels as it dissipates is referred to as its path of migration.

The combustible range for natural gas is from $5 \%$ to $15 \%$ by volume. Gas concentrations at the center of a release are typically too rich (above the 15\% concentration) to support combustion. However, as the gas disperses, the concentration diminishes to a fuel/air ratio that is capable of combustion. As dispersion continues, the concentration decreases to where it becomes too lean (below 5\% concentration) and is no longer combustible. During the initial release, it is not uncommon to have natural gas concentrations within the combustible range until the concentration diminishes over time.

Unlike gasoline or diesel vapors, the ignition of natural gas can only occur when concentration levels are within the 5\%-15\% range.

Natural gas can be ignited by a spark, flame, or very hot surface. To eliminate the hazard of ignition, a maintenance facility must be modified to eliminate potential ignition sources that may come into contact with released gas. Additionally, a maintenance facility must be modified to ensure that the air circulation exhausts gas as quickly as possible to minimize the amount of time that gas concentrations are within the combustible range.
The inadvertent release of natural gas can occur in several ways. For example, a vehicle may have a slow leak due to an improperly sealed fuel system component. Such minor releases may also occur when disconnecting fuel system components during a repair such as a fuel filter change. In general, it is unlikely that combustible concentrations of natural gas will result from minor leaks that occur over time because the natural gas released from these types of leaks will dissipate rather quickly.

Though rare, a creditable release of natural gas may occur if a CNG tank or fitting fails resulting in the entire volume of the tank emptying rapidly. To prevent against creditable releases, CNG tanks are equipped with a pressure relief device that is set to open in the event of excessive pressure or temperature, releasing smaller and more controlled volumes of natural gas and allowing the vehicle system and tank pressure to return to a safe range.

A facility must be modified to eliminate potential ignition sources that may come into contact with released gas, as well as to ensure that the air circulation exhausts gas as quickly as possible.

While a CNG maintenance facility is designed to safely handle all types of releases, it is the "creditable" uncontrolled release that presents the greatest danger. Although this type of event is extremely rare, a maintenance facility must be designed to protect against it.

Because natural gas is lighter than air, released gas is likely to experience interruptive forces as it disperses throughout the structure. Paths of migration are also affected by air movement within the structure. For this reason, a maintenance facility must be designed to prevent released gas from entering unprotected areas as it rises and disperses. This is accomplished by installing
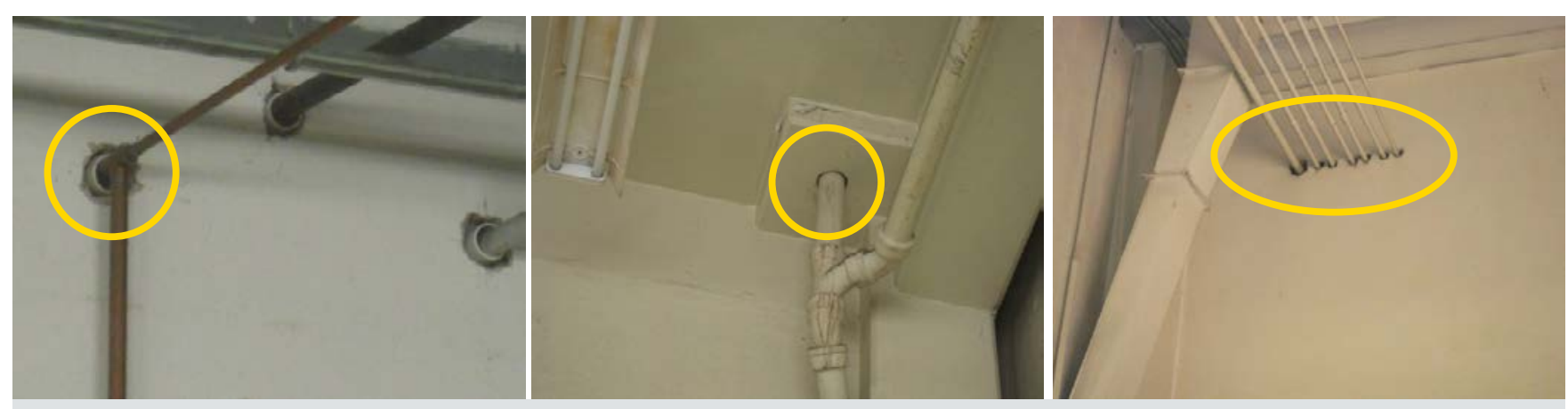

Figure 1. Unsealed conduit or pipes passing through walls or ceilings present a potential path of migration. Photos courtesy of Gladstein, Neandross \& Associates, NREL 44893, 44894, and 44895 

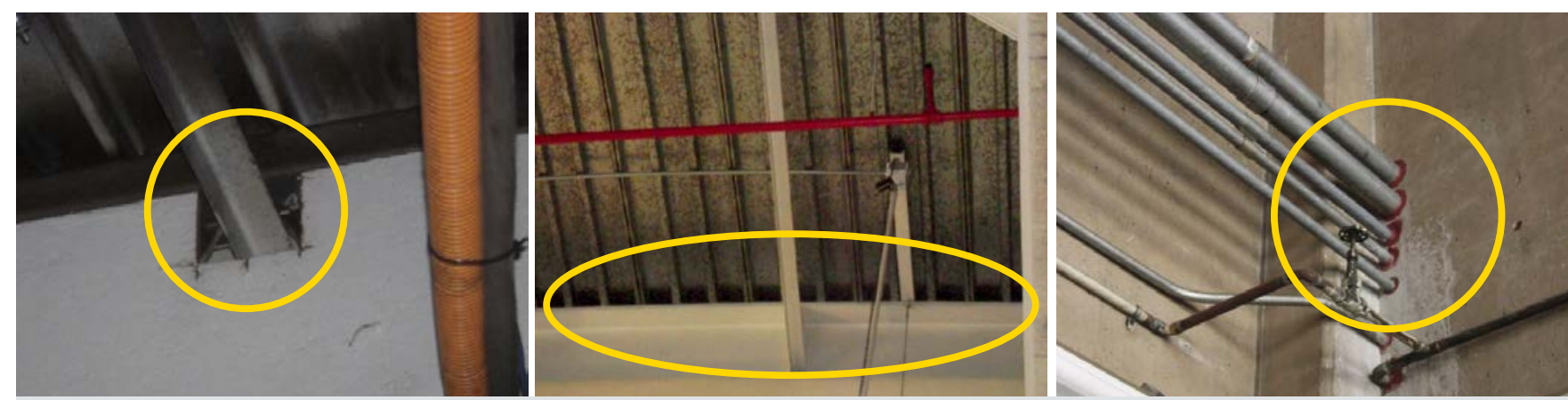

Figure 2 (left). Structural members passing through a wall present a potential path of migration.

Figure 3 (middle). Gaps between walls and the corrugated ceiling panels present a potential path of migration.

Figure 4 (right). Wall penetrations properly sealed to prevent migration. Photos courtesy of Gladstein, Neandross \& Associates,

NREL 44897, 44898, and 44896

barriers, pressurizing adjacent areas, and sealing gaps in the structure to prevent migration into unprotected areas.

Examples of potential paths of migration resulting from the structural characteristics of a facility are shown in Figures 1-3. These potential paths of migration can be eliminated by installing sealing material around the wall penetrations and at the ceiling level, as shown in Figure 4.

Figure 5 shows an open passage to an adjacent nonmaintenance area through which gas migration could occur. The two areas must be isolated to prevent migration between the two rooms.

Stairways accessing an upper level could accentuate the upward gas flow (Figure 6). In these cases, the potential path of migration should be intercepted by isolating the two levels to prevent natural gas from entering an unprotected area. One method for isolating two areas is to install a self-closing door between them. The door must be fitted with a self-closing mechanism to keep it closed except when in use. Operating protocols and signage should also be used to ensure that the door is not propped open. Doors should not be fitted with louvers or other means of ventilation that may create paths of migration.

Figure 7 shows a vehicle parts room adjacent to a maintenance area that is serviced with a pass-through window-a potential path of migration to an unprotected area. This hazard can be eliminated by permanently sealing the window, adopting strict operating protocols to ensure it is only open during active use, or installing a pressurizing fan in the parts room to prevent the inward flow of gas from the maintenance area.

\section{Ventilation}

Ventilation of a CNG vehicle maintenance facility is one key area of concern that is addressed by the applicable codes. Proper ventilation can be achieved by mechanical means (powered fans) or by convection to provide air flow. However, it is rare that convection alone can provide sufficient or predictable air flow. There are several reasons
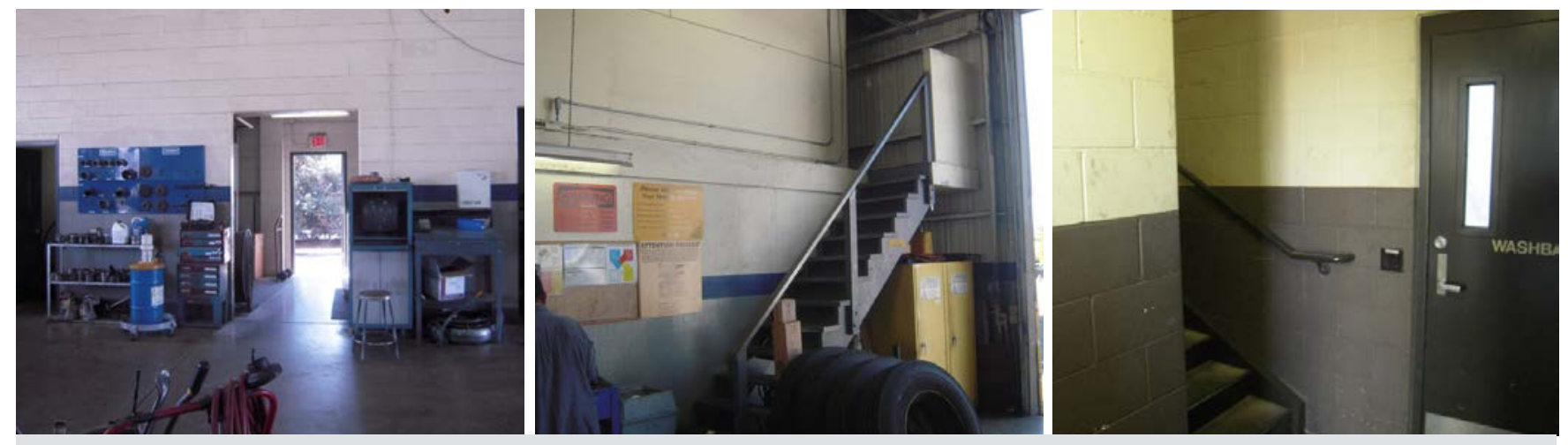

Figure 5 (left). A potential path of migration created by an open passageway.

Figure 6 (middle, right). Examples of stairways to upper levels that are potential paths of migration. Photos courtesy of Gladstein, Neandross \& Associates, NREL 44899, 44900, 44901 


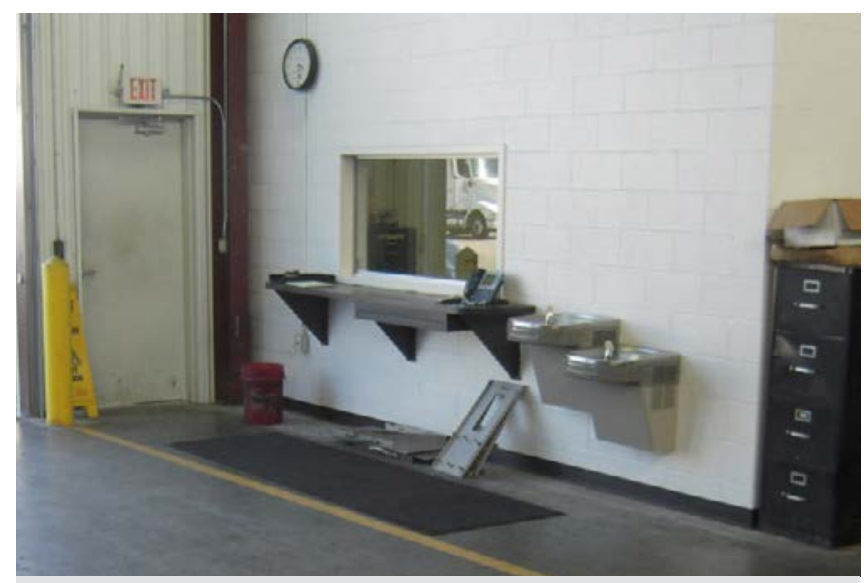

Figure 7. A pass-through window presents a potential path of migration. Photo courtesy of Gladstein, Neandross \& Associates, NREL 44902

for ventilating a maintenance facility. Naturally, ventilation is necessary to quickly and effectively dilute released natural gas so that the gas concentration is below the combustible level. However, ventilation is also necessary to direct gas to the detection and alarm systems that will alert occupants to safely evacuate the facility. Lastly, ventilation aids in preventing the gas from accumulating near potential ignition sources.

The IFC and NFPA differ in their calculations and control strategies. Knowing which code requirements the local governing body follows is critical to determining which control strategy should be implemented.

Unfortunately, ventilation volumes and strategies are one area where the codes differ significantly. All agree that natural ventilation may be allowed at the discretion of the AHJ. However, if the AHJ elects to require mechanical ventilation, the IFC and NFPA differ in their calculations and control strategies. Knowing which code requirements the local governing body follows is critical to determining which control strategy should be implemented.

Ventilation is necessary to quickly and effectively dilute released natural gas. It is also necessary to direct gas to the detection and alarm systems and prevent it from accumulating near potential ignition sources.

\section{IFC Ventilation Requirements}

The IFC states that major repair garages shall be ventilated at a rate of one cubic foot per minute (cfm) for every 12 cubic feet of structural space, which is equivalent to five air changes per hour (ACH). Note that this calculation is based on the total volume of the structure, not the square footage of the floor or ceiling. Furthermore, the IFC provides three options for controlling ventilation: 1) continuously, 2) continuously while the space is occupied via interconnection with the lighting circuit, or 3) upon demand for dilution ventilation triggered by the gas detection system.

The potential energy impacts of continuous operation and tempering of the entire volume of air in a structure influences the design of many maintenance facilities, particularly those in cold climates. For this reason, a maintenance facility manager may elect to use methane detection as the triggering mechanism instead of continuous ventilation. This method incurs similar installation costs but results in greatly reduced operating costs. Various approaches to ventilation system design and installation of methane detection systems are discussed later in this section.

\section{NFPA 30A Ventilation Requirements}

Ventilation effectiveness under NFPA 30A is also based on the total volume of the structure. However, it focuses on potential electrical sources of ignition within the area 18 inches below the ceiling. This area is classified as Class I, Division 2. NFPA 30A states that electrical devices located in this zone do not need to meet Class I, Division 2 specification if the structure has an effective continuous ventilation rate of at least $4 \mathrm{ACH}$. If the structure does not have this continuous ventilation rate, existing electrical devices that do not meet the Class I, Division 2 specification must be relocated to below this 18-inch zone or replaced with electrical devices rated for Class I, Division 2 service.

NFPA 30A does not specifically address operational parameters of ventilation systems in major repair garages. Generally, fire marshals and other AHJs tend to refer to the IFC operational guidelines of 1) continuous operation, 2) continuous operations while occupied, via interlock with lighting, or 3) methane detection system-triggered. If the third operational parameter is selected, then all electrical devices in the "ceiling zone" must be removed, relocated, or replaced with devices rated for Class I, Division 2 applications. 


\section{"Hybrid" Application of IFC and NFPA}

Most local governing bodies will require major repair garages to meet the most stringent requirement, which requires the ventilation system to be sized for $5 \mathrm{ACH}$. However, it is essential to ensure that at the 5-ACH ventilation rate the building design and construction do not potentially hinder effective air changes in the ceiling zone. Mechanical ventilation strategies should be reviewed in advance with the AHJ to ensure that effective ventilation is achieved in the ceiling zone.

\section{Ventilation Strategies}

The applicable codes do not specifically address how the ventilation is to be achieved. Most facilities rely on exhaust fans to move air out of the facility. In this case, make-up air is provided via natural draft through openings such as doors and louvers, or by mechanical means such as make-up air units. Facilities that were not designed with rooftop exhaust fans instead feed make-up air into the space with interlocked electronically actuated gravity vents in the ceiling to maintain proper air balance. The codes require that the exhaust be through the roof of the structure and that makeup air be provided from near floor level.

A variety of approaches are available, each with advantages or disadvantages based on factors such as 1) the local governing body's decision regarding applicable code, 2) the design and construction of the existing facility and its ability to accommodate modifications, 3) the climate in which the facility is located (i.e., warm weather versus cold weather and related air-tempering energy costs), 4) ease of operation, and 5) cost.

Ventilation approaches are based on factors such as:

1. The local governing body's decision regarding applicable code

2. The design and construction of the existing facility and its ability to accommodate modifications

3. The climate in which the facility is located

4. Ease of operation

5. Cost.

The most common form of facility ventilation is a roofmounted upblast fan that vents into the atmosphere (Figure 8). These exhaust fans can pull air directly from the space via the fan inlet as shown in Figure 9, or they

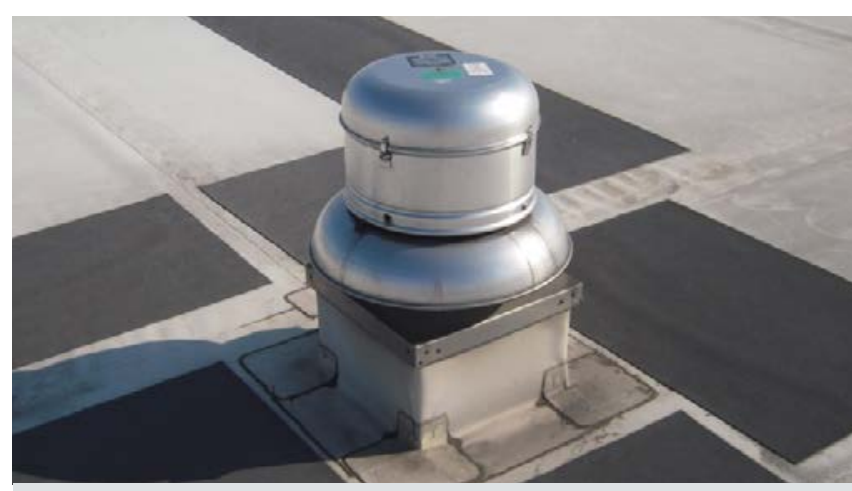

Figure 8. Roof-mounted upblast fan. Photo courtesy of Gladstein, Neandross \& Associates, NREL 44698

can pull air through exhaust ductwork serving specific areas of the ceiling zone as shown in Figure 10.

Most existing maintenance facilities are equipped with roof-mounted fans, some of which may be rated for use in Class I, Division 2 areas. In some cases, existing roofmounted fans can be used with minimal modifications for compliance with CNG vehicle maintenance facility requirements. Roof-mounted fans require certain characteristics to be suitable for use in a CNG vehicle maintenance facility. For example, the fan blades and shrouds must be constructed from non-sparking materials

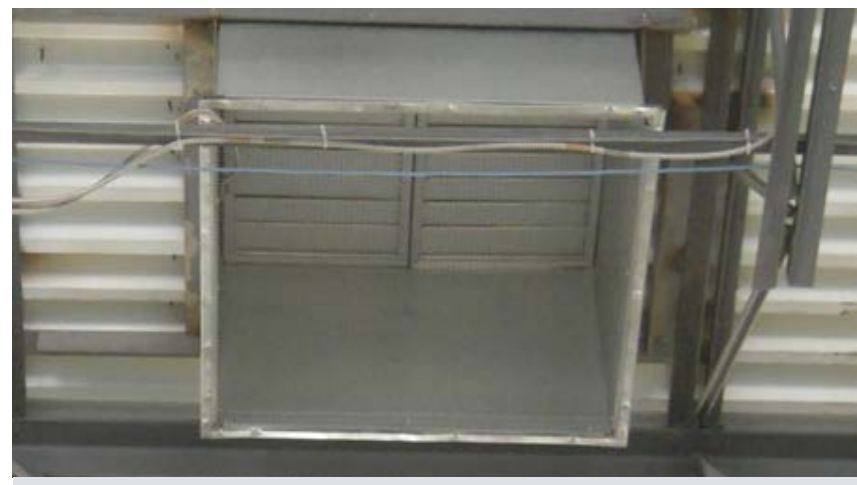

Figure 9. Direct fan intake with louvers. Photo courtesy of Gladstein, Neandross \& Associates, NREL 44700

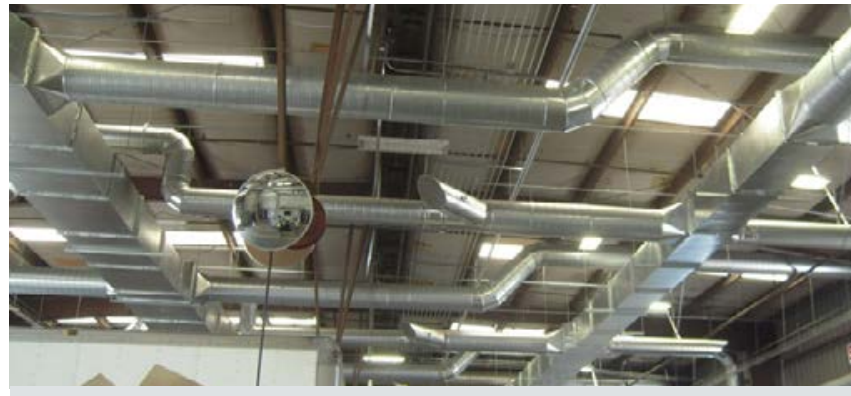

Figure 10. Intake ducting used to evacuate air from specific areas. Photo courtesy of Gladstein, Neandross \& Associates, NREL 44701 


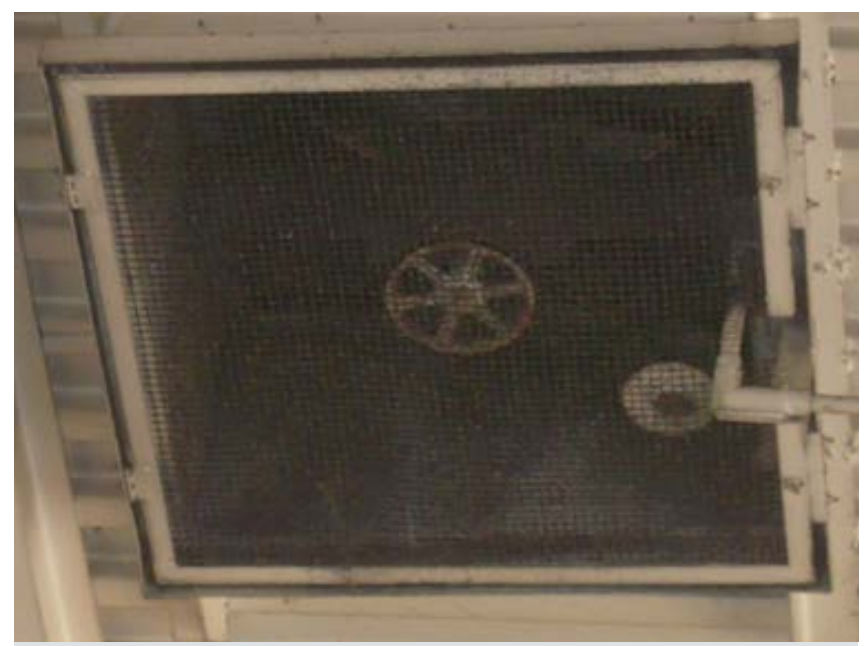

Figure 11. Non-compliant fan has fan intake flowing over the motor. Photo courtesy of Gladstein, Neandross \& Associates, NREL 44702

(i.e., plastic or aluminum) to prevent the potential for igniting released gas. In addition, the electric motor that drives the fan must be explosion-proof or located where the fan intake or exhaust does not pass over the electric motor. Figure 11 shows a non-compliant belt-driven fan with the fan intake flowing over the electric motor. Certification of compliance must be obtained from the manufacturer, and the type of fan selected must be approved by the AHJ.

In some cases, there is not enough space to install a fan or the roof cannot support the additional load of a new fan. In these cases, ground-mounted fans and suitable external ducting (Figure 12) can be used to comply with requirements.

Operation and control of the ventilation system depend on several factors. The exhaust fans and means for providing makeup air can 1) operate continuously, 2) be controlled automatically using a gas-detection system, or 3 ) be activated through the facility overhead and workspace lighting system. A description of each method for operation and control of the ventilation system follows:

1. For ventilation systems that operate continuously, the fans are constantly running. Continuously operating ventilation systems are most commonly employed in warmer climates where ambient air does not require conditioning for worker comfort. These systems require continuous electrical power for the fans and may entail increased fan maintenance costs.

2. For systems controlled using a combustible-gasdetection system, the fans are only activated when the sensors detect the presence of natural gas

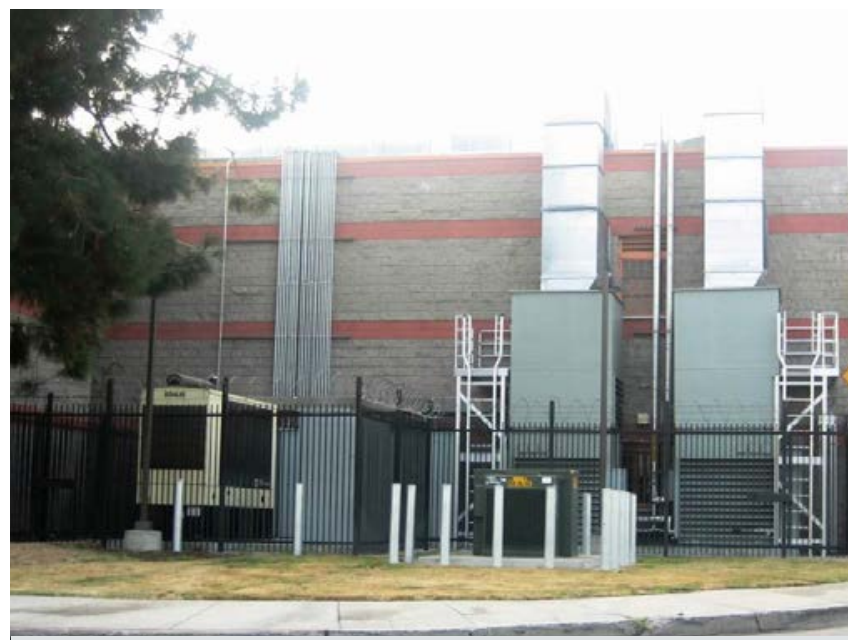

Figure 12. Ground-mounted fan with external ducting. Photo courtesy of Gladstein, Neandross \& Associates, NREL 44703

(manual operation is also possible). Another important consideration is that if non-rated electrical equipment is within 18 inches of the ceiling and protection is provided by ventilation only (at a rate of $4 \mathrm{ACH}$ ), the fans must be in continuous operation when $\mathrm{CNG}$ vehicles are in the building.

3. For systems activated in conjunction with facility lighting, the ventilation system is interlocked with the lighting circuit. The applicable codes and regulations assume that interior lighting will always be activated whenever a maintenance facility is occupied. Similar to the continuous ventilation system, the associated high heating costs may make this type of system cost prohibitive in colder climates. This option should also be implemented with care, as a release could occur when the building is not occupied and the lights are not in use. Also note that the ventilation required consists of $100 \%$ make-up air with no recirculation. Therefore, although this option is compliant with some codes, it is not recommended.

Some designers recommend that two levels of ventilation be employed by utilizing two-speed or variablespeed fan motors. A two-level system will provide a lower level of ventilation (4 ACH) to reduce noise levels and energy consumption while the second stage will provide rapid dilution. The approach taken must be approved by the AHJ.

Facility ceiling profiles vary considerably as a result of structural roof members, varying ceiling heights, or other internal configurations. The codes do not require airflow across every square inch of ceiling; rather, the system should be designed such that the airflow eliminates 
accumulations of natural gas which may be subject to ignition sources. Figure 13 shows a ceiling configuration that could accumulate concentrations of natural gas. Because the "pockets" do not contain heaters, electrical wiring, or appliances, there is no ignition source and it is not necessary to ventilate each "pocket."

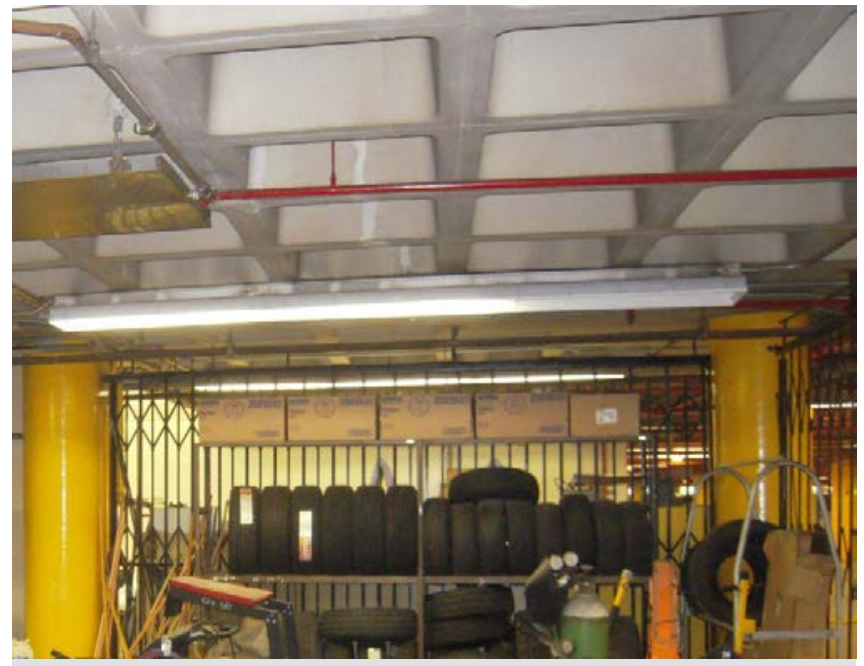

Figure 13. Ceiling configuration with "pockets." Photo courtesy of Gladstein, Neandross \& Associates, NREL 44704

Make-up air for the ventilation system may be derived from a number of sources. In warm climates, makeup air may be supplied through open bay doors. In cool climates or in hot climates where cooling is provided, a continuous ducted ventilation system may be employed. In the case of ducted systems, the bay doors may be opened by the gas detection system interlock to provide additional makeup air flow and additional paths of personnel egress. It is not necessary to open all of the bay doors in a multi-bay maintenance facility, only a sufficient number to provide the required makeup air flow.

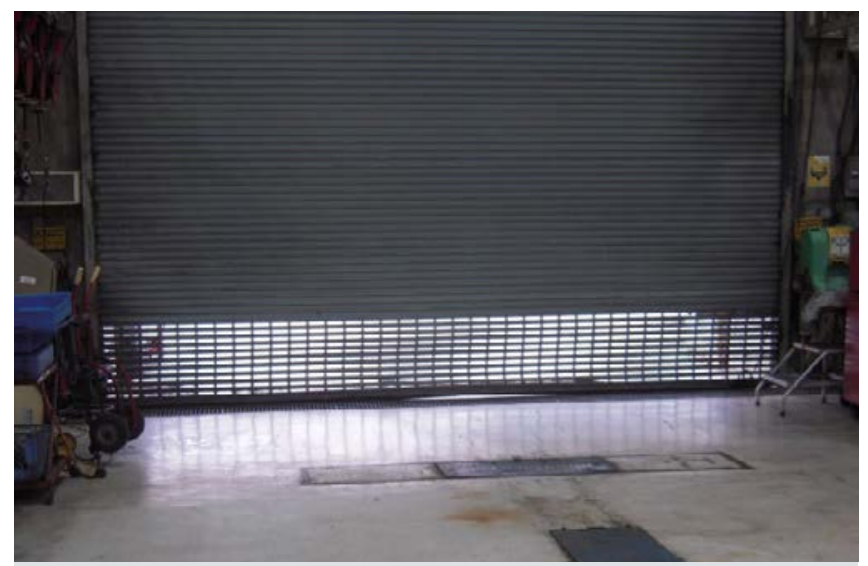

Figure 14. Security mesh in overhead doors. Photo courtesy of Gladstein, Neandross \& Associates, NREL 44705
Some maintenance facilities have bay doors equipped with stops that maintain a gap (typically around 12 inches), providing a permanent ventilation space between the bottom of the door and the ground when fully "closed." If this type of configuration presents a security concern, a mesh or a grate can be installed at the bottom of the door as shown in Figure 14.

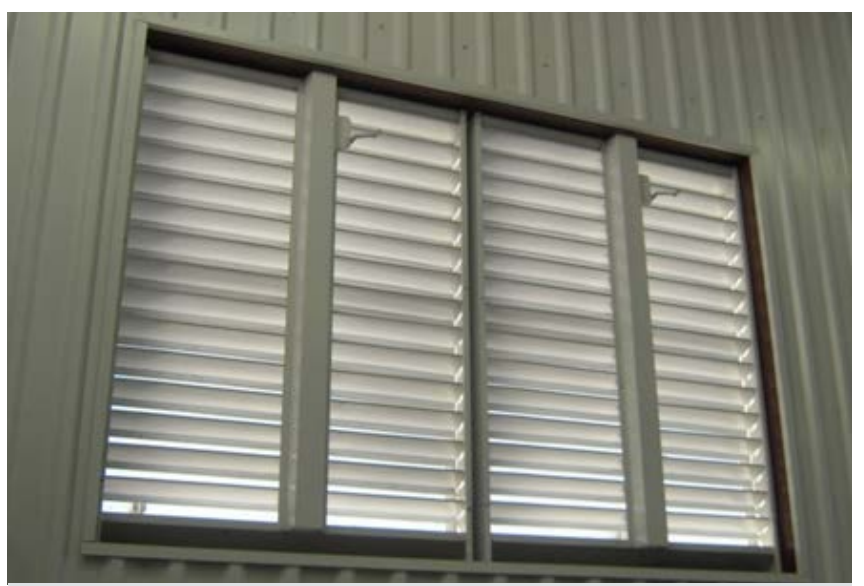

Figure 15. In-wall louver that provides make-up air flow. Photo courtesy of Gladstein, Neandross \& Associates, NREL 44706

In-wall louvers (Figure 15) can also be employed to provide a portion of the makeup air flow. Generally, they are not able to provide the sufficient make-up air volume or flow direction to meet the codes and regulations on their own. Powered side wall fans (Figure 16) may also be used to provide the requisite air flow.

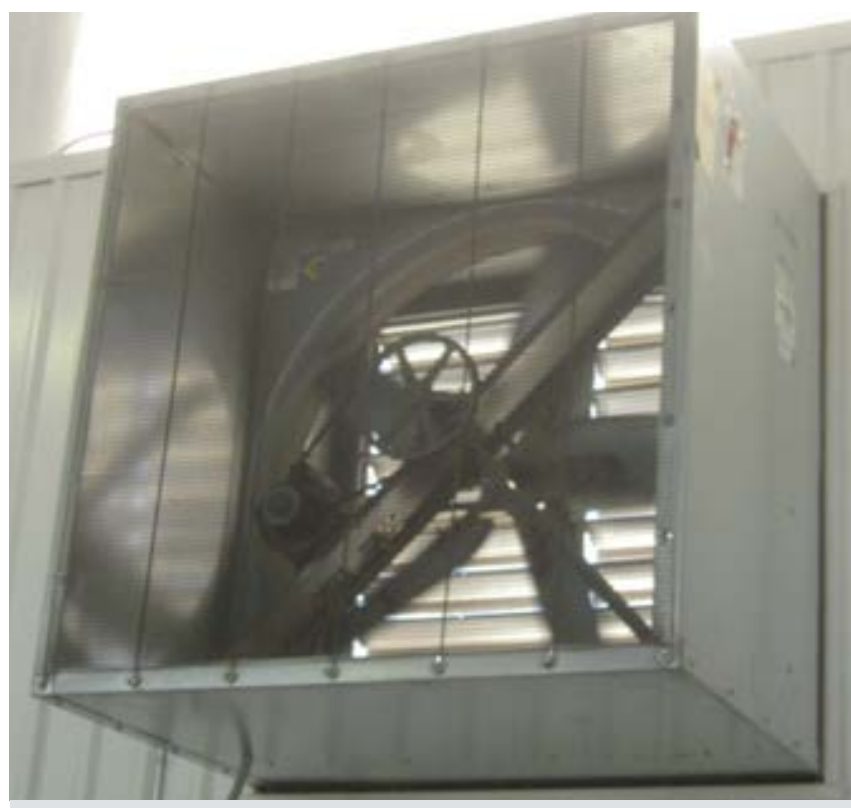

Figure 16. Powered sidewall fan that provides make-up air flow. Photo from Gladstein, Neandross \& Associates, NREL 44707 
Existing maintenance facilities designed to service conventionally fueled (gasoline and diesel) vehicles are typically fitted with ventilation systems that capture vapors in the event of a spill. Because fuel vapors are heavier than air, the ventilation system pickup point is typically located near the floor of the facility (Figure 17) where the vapors will accumulate. A maintenance facility that services both conventional and $\mathrm{CNG}$ vehicles needs ventilation systems to address conventional fuel vapors as well as natural gas releases. An existing facility that is modified to service $\mathrm{CNG}$ vehicles must retain the ventilation

\section{A facility that services both conventional and CNG} vehicles needs ventilation systems to address conventional fuel vapors as well as natural gas releases.

system that was designed to manage conventional fuel vapors or must provide liquid fuel code compliance in the design of the new ventilation system. Rare exceptions may be granted by the AHJ to allow the existing ventilation fans to be used as long as they are compliant with natural gas use and equipped with automatic dampers to close off the flow from floor level and open the pickup points at the ceiling level.

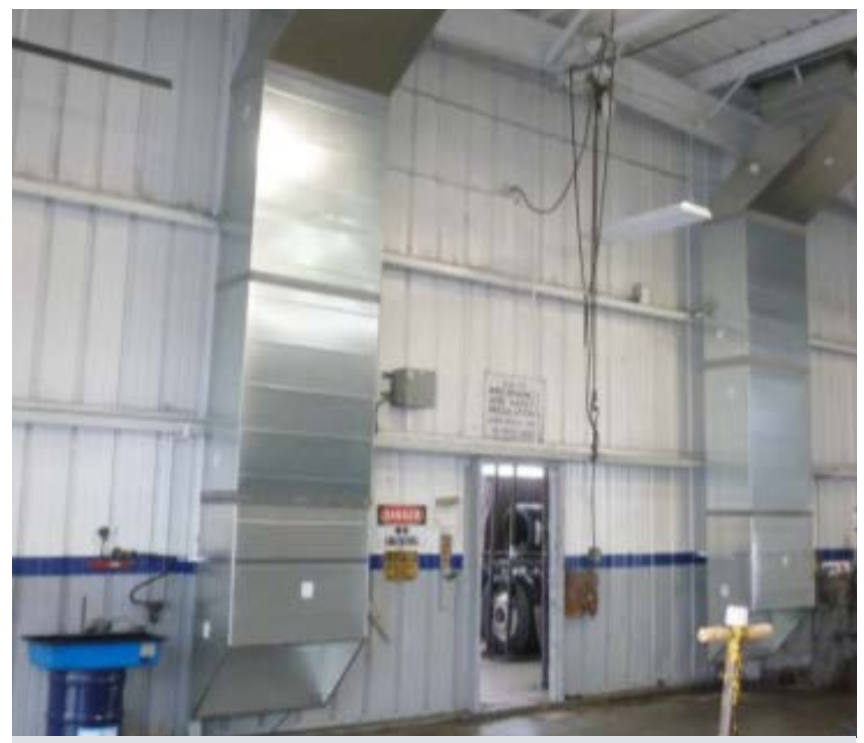

Figure 17. Conventional fuel maintenance facility ventilation pickup points. Photo courtesy of Gladstein, Neandross \& Associates, NREL 44699

Regardless of the ventilation system selected, it must be designed by a registered/licensed engineer. Calculations showing the volume of air moved are required on the final design drawings.

\section{Space Heating}

Maintenance facilities in most locations require some form of space heating during the winter months to ensure worker comfort. Heat may be provided using a forced air central heating system that warms and circulates the air throughout the maintenance facility or utilizes an air-to-air heat exchanger. Localized space heating is another method for heating a maintenance facility. Localized space heating is typically accomplished using gas-fired infrared direct radiation or tube-type heaters, gas- or propane-fired devices equipped with fans, or electric infrared heating units. Most of these types of heating apparatus are either mounted to the walls of the facility or suspended from the ceiling. Portable heaters used to provide supplemental heat in specific areas are typically placed on the floor.

Codes and regulations require that specific conditions be met for both heaters and heating systems. Classification of the facility as either a major repair or minor repair category is also an important factor.

NFPA 30A: Heating equipment with open flames or exposed surfaces with a temperature above $750^{\circ} \mathrm{F}$ cannot be placed in areas that may experience a creditable release. This includes all heating devices in a major repair garage.

For major repair garages, NFPA 30A states that heating equipment with open flames or exposed surfaces that have a temperature in excess of $750^{\circ} \mathrm{F}$ shall not be permitted in areas that may experience a creditable release. For practical reasons, all heating devices in a major repair garage are subject to this limitation. Although not specifically stated, these same concerns and design approaches are also generally applied to minor repair garages.

Most existing heating systems, except forced-air types, present specific challenges for CNG vehicle maintenance facility compliance. For flame-fired heaters, the flame itself is an ignition source. For gas-fired infrared direct radiation heaters, even for those where the flame is contained within the heating elements, the surface temperature of the radiant heating elements that exceed $750^{\circ} \mathrm{F}$ present a potential ignition source. Electric heaters that employ heating coils and a fan may or may not be compliant depending upon the operating temperatures reached by the heating coils. Figures $18-21$ depict various types of space heating equipment commonly found in maintenance facilities that would need to be replaced, modified, or eliminated. 


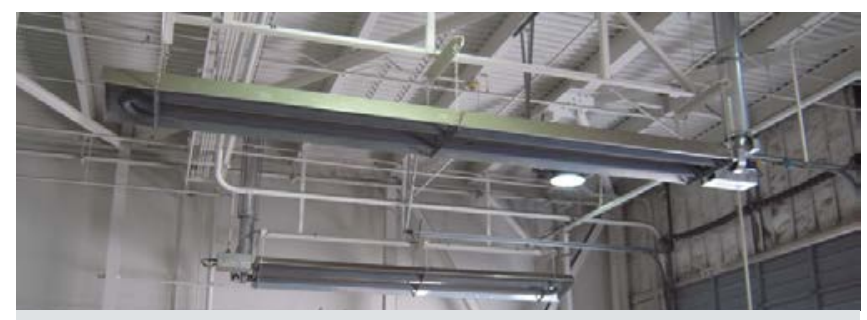

Figure 18. Gas-fired infrared tube-type heater needs to be replaced or removed. Photo from Gladstein, Neandross \& Associates, NREL 44903

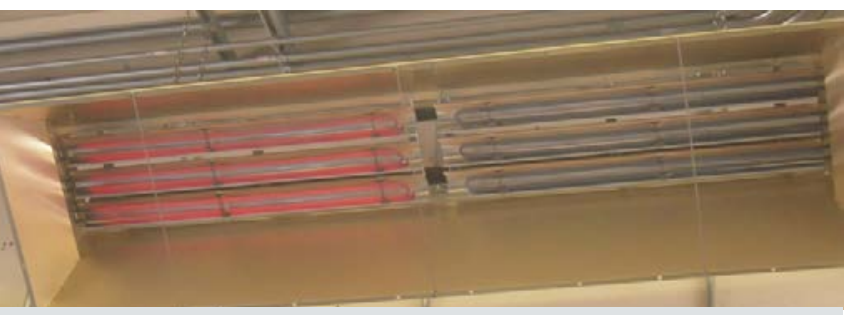

Figure 19. Electric infrared radiant heater needs to be replaced or removed. Photo from Gladstein, Neandross \& Associates, NREL 44904

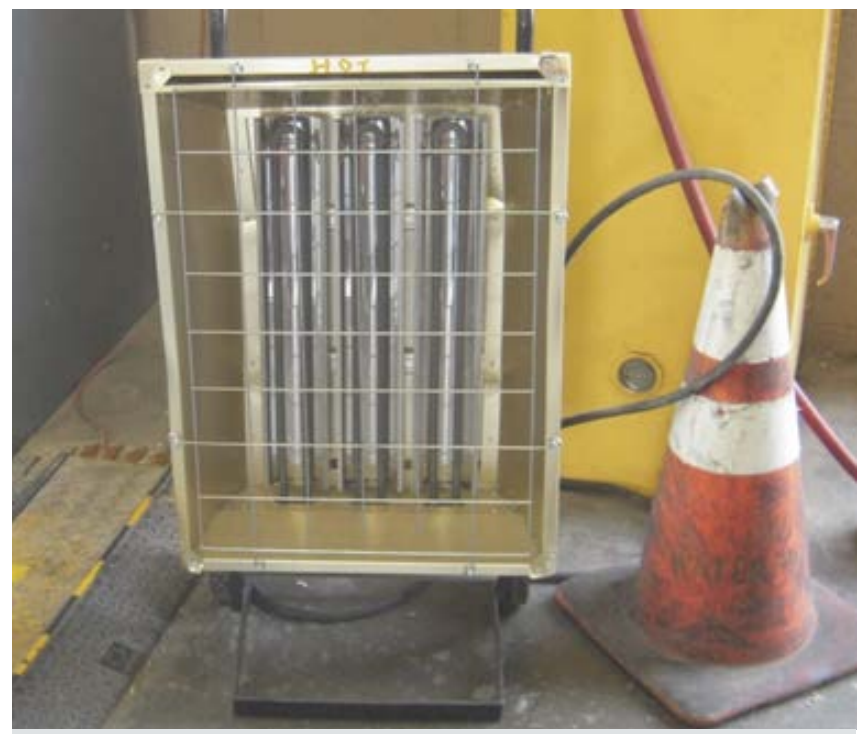

Figure 20. Floor-mounted portable propane heater should be eliminated. Photo from Gladstein, Neandross \& Associates, NREL 44905

Compliance with the codes and regulations can be achieved by simply removing the non-compliant heating units and/or replacing them with units that are considered compliant. Gas-fired infrared tube-type radiant heaters (Figure 18) are commonly employed in maintenance facilities that service conventionally fueled vehicles. Manufacturers of these types of heating systems now produce units that have contained flames and surface elements that do not exceed $750^{\circ} \mathrm{F}$. In this

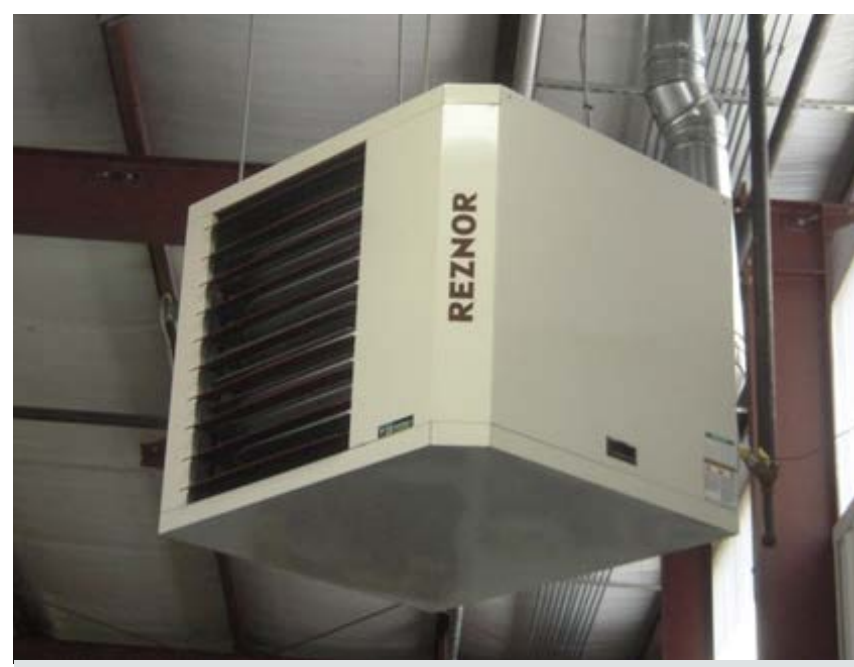

Figure 21. Gas-fired fan heater needs to be replaced or removed. Photo from Gladstein, Neandross \& Associates, NREL 44696

instance, modifying the heating system to be compliant with the codes would only require replacing the heater(s). However, it is essential that the supply air and exhaust flow from these heater(s) be completely contained and directed from and to the outside of the facility. This can be accomplished by routing the inlet air and exhaust air through the facility wall or roof.

Portable propane heaters (Figure 20) are prohibited from use within a CNG vehicle maintenance facility. Gas-fired fan-type heaters (Figure 21) are not compliant because of their inherent open flame(s) and must be replaced with units that meet the codes. This includes gas-fired fan-type heaters in which the only open flame is the pilot light.

Although forced air heating systems do not present direct hazards as potential ignition sources for combustible gas, modifications may be required to their air flow systems because of their potential effect on the paths of migration. However, such continuous ventilation will extract warmed air from the building, and such single-pass heating may be cost prohibitive, particularly in colder climates. To address this heat loss, many owners utilize units with heat recovery. Ducted heating units should be designed to provide air flow prior to igniting a burner.

\section{Electrical Wiring, Lighting, and Appliance Considerations}

Electrical wiring, lighting, and electrical appliances all present potential ignition sources within a maintenance facility. A spark that is caused by an abnormal condition such as a short circuit within a wire conduit or junction 
box or generated in normal operation of an unprotected electric motor is cause for concern. Additionally, unsealed conduits may also provide a means of gas migration from one portion of the facility to another. Before modifying a maintenance facility to service $\mathrm{CNG}$ fuel vehicles, the existing design must be taken into consideration so that each of the safety risks is mitigated.

NFPA 30A states that in major repair garages, the area within $18 \mathrm{in.} \mathrm{of} \mathrm{the} \mathrm{ceiling} \mathrm{is} \mathrm{considered} \mathrm{a} \mathrm{hazardous}$ zone and is designated as Class I, Division 2. Within these areas, all electrical wiring, conduits, junction boxes, and electrical appliances must be either explosion proof or relocated outside the hazard zone. In general, it is less expensive to eliminate or relocate non-compliant electrical fixtures than to replace them with more costly explosion-proof units. The NFPA 30A Class I, Division 2 designation does not apply for minor repair garages. However, it is best practice to also eliminate credible sparking risks in minor repair garages.

In the hazardous zone within 18 in. of the ceiling, all electrical wiring, conduits, junction boxes, and electrical appliances must be either explosion proof or relocated outside the zone.

It is important to note that NFPA 30A does provide an exception to the Class I, Division 2 designation. NFPA $30 \mathrm{~A}$ states that if the structure has an effective continuous ventilation rate of at least $4 \mathrm{ACH}$, the area within 18 inches of the ceiling is not considered a hazardous zone. This exception is covered in Section 3.2, Ventilation.

For maintenance facilities where providing continuous ventilation is deemed impractical, the following provides solutions for many commonly encountered conditions.

In some cases, the number of conduits or junction boxes located in the hazardous zone is large enough that relocating each of them is unreasonable because of the cost or complexity (Figure 22). Class I, Division 2 compliance can be obtained if conduits or junction boxes are provided with seal-offs that create a physical barrier that prevents gases from traveling freely through the conduit as well as the migration of methane into the conduits. The use of seal-offs in lieu of relocation must be approved by the AHJ.

According to NFPA 30A, low-voltage wiring and containing conduits may be exempt from the Class I, Division 2 requirements if they are determined to be

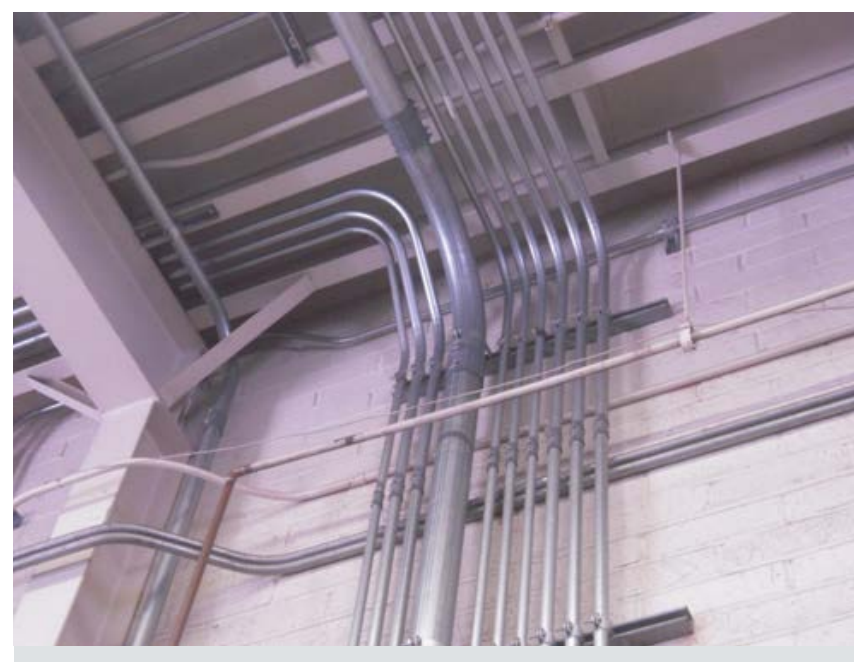

Figure 22. Relocating multiple conduits, such as those shown here, would be costly and complex. Photo from Gladstein, Neandross \& Associates, NREL 44676

non-sparking. Examples may include loudspeaker and security camera wiring; wiring used for data transmission; alarms; and wiring used for similar applications (Figure 23). However, the AHJ must approve the lowvoltage wiring; otherwise, it may be considered hazardous and subject to Class I, Division 2 requirements.

For lighting fixtures to comply with Class I, Division 2 requirements, they must be either classified as explosion-proof or moved to a location that is more than 18 in. from the ceiling. All components (e.g., fixtures, bulbs, ballasts, transformers, etc.) must be relocated outside of the hazardous zone (Figure 24). In general, lowering the fixtures and components is the most common and cost-effective approach for compliance.

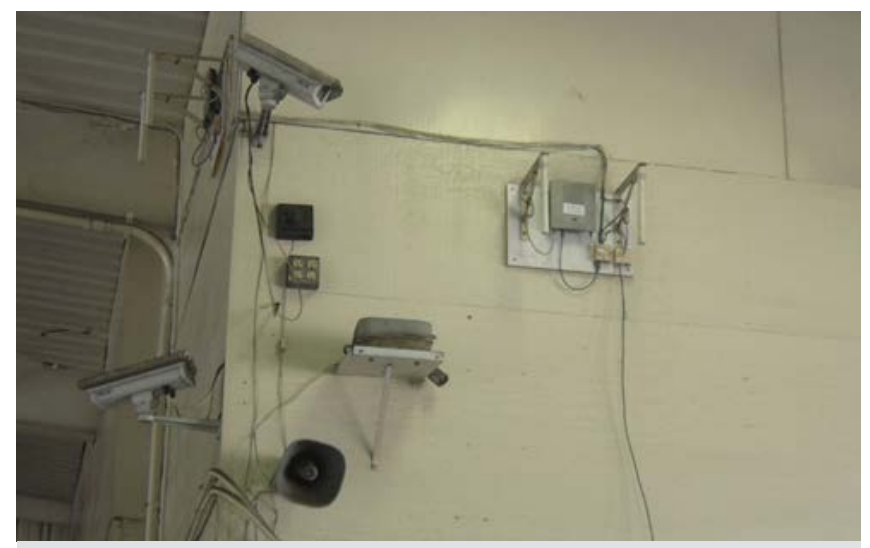

Figure 23. Low-voltage wiring and devices. Photo from Gladstein, Neandross \& Associates, NREL 44677 


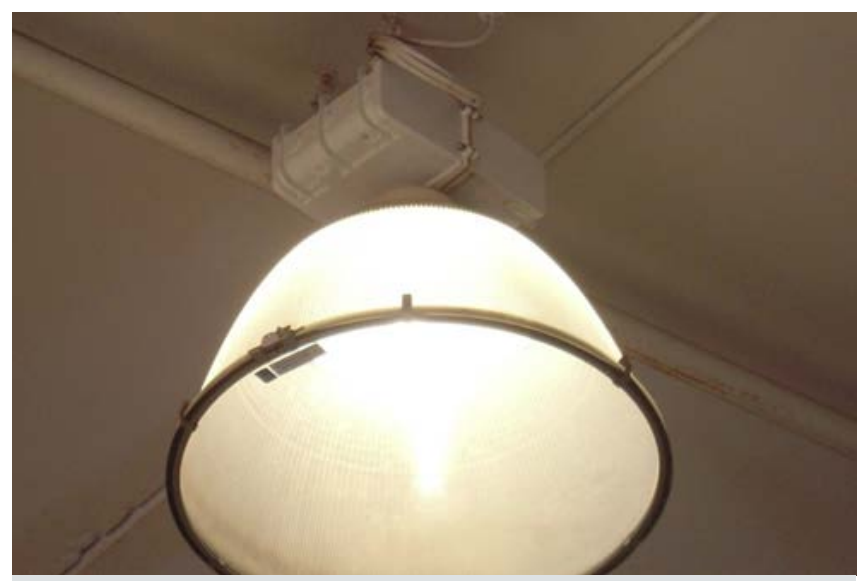

Figure 24. Overhead lighting fixture and ballast that must be relocated. Photo from Gladstein, Neandross \& Associates, NREL 44678

Other electrical appliances and equipment within the hazardous zone must also be removed or relocated. Figure 25 displays a typical rotary fan that, although not located within 18 in. of the ceiling, would be determined as a potential ignition source because of the possibility that sparks may be generated within the drive motor. The rotary fan would be classified as a potential ignition source because rising released gas could encounter the fan before reaching the ceiling-mounted gas detectors. In some cases, the AHJ may require that all such fixtures be removed completely to avoid this hazard.

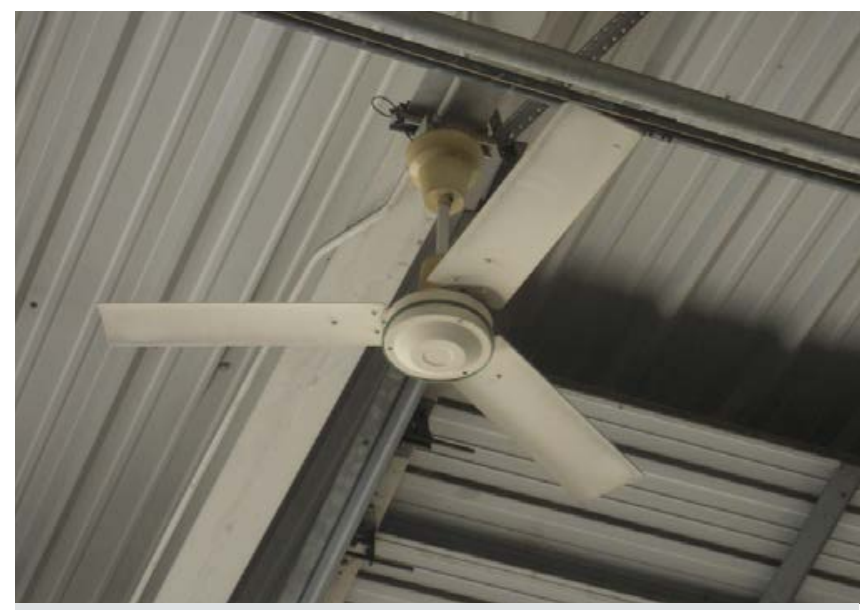

Figure 25. An overhead rotary fan that presents a potential ignition source. Photo from Gladstein, Neandross \& Associates, NREL 44679

Electric door motors, commonly found on main bay doors, are also a potential ignition source even when they are not located within the hazardous zone (Figure

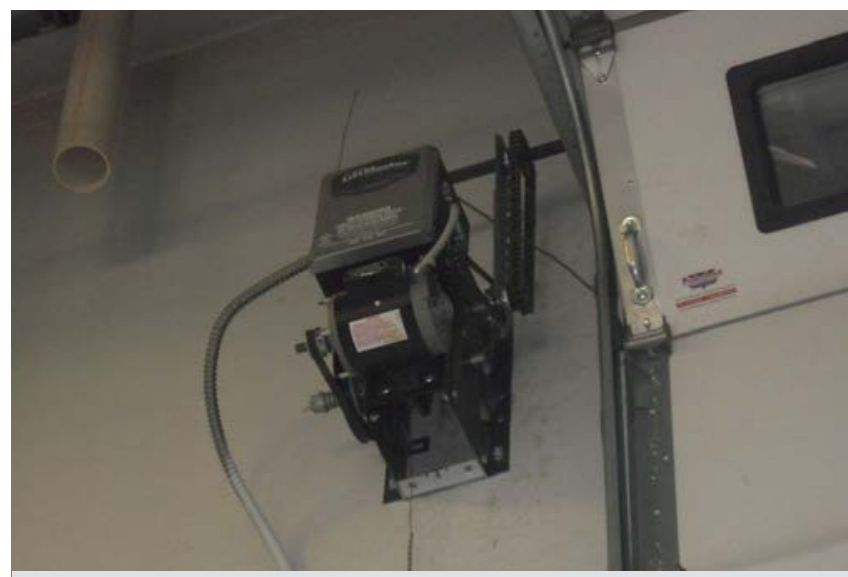

Figure 26. A typical electric door motor. Photo from Gladstein, Neandross \& Associates, NREL 44680

26) because of the possibility that sparks may be generated by the electric motors. If gas is detected, the electric door motors will be activated to open the doors. For this reason, depending on the type of motors, the accompanying proximity switches, and the physical location of

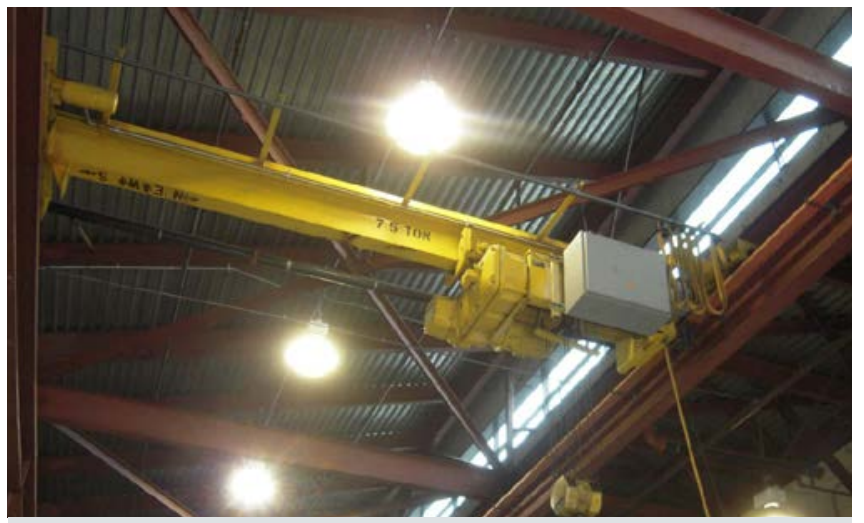

Figure 27. A typical overhead crane. Photo from Gladstein, Neandross \& Associates, NREL 44681

the openers, AHJs may require electric door motors to be upgraded or replaced with explosion-proof equipment. Doors that are not activated during a gas detection event do not need to be replaced with explosion-proof equipment. Similarly, overhead cranes (Figure 27) should be shunt-tripped to prevent activation of their electric motors in the event of a gas release. Shunt tripping, which is further discussed in Section 3.5, Gas Detection, Alarm, and Control Systems, involves an interface between the methane detection controls and the circuits within the facility electrical panel. When methane is detected, selected circuits can be instantaneously deactivated. 
A gas detection and alarm system is strongly recommended for a number of reasons. It greatly enhances personnel safety and property protection, and the facility's insurance underwriter may require the installation of such a system.

\section{Gas Detection, Alarm, and Control Systems}

The applicable codes and regulations require that any facility that maintains vehicles that use non-odorized gas fuel be equipped with a flammable gas detection system. For example, NFPA 30A includes the following requirements:

- The flammable gas detection system must be calibrated to the type of fuel being used, and the system must be activated when a concentration of $25 \%$ of the LFL is detected.

- Upon gas detection, the system must activate audible and visual alarms, deactivate the heating system(s) and turn on the ventilation system.

- If a failure occurs within the gas detection system, the system must automatically deactivate the heating system(s), activate the ventilation system, and activate an audible trouble signal in a location approved by the AHJ.

A combustible gas detection and alarm system will:

- Provide early warning to occupants

- Initiate actions to eliminate potential ignition sources

- Initiate actions to promote quick dilution of the gas.

The applicable codes suggest, but do not categorically state, that a gas detection system is not required for a facility that maintains vehicles that use odorized gas fuel such as CNG. But industry experience has shown that AHJs generally require a gas detection system despite the apparent exemption within NFPA 30A. A gas detection and alarm system is strongly recommended for a number of reasons. It greatly enhances personnel safety as well as property protection. In addition, the facility's insurance underwriter may require the installation of such a system.
The primary functions of a combustible gas detection and alarm system are to:

- Provide early warning to occupants that a methane gas release has occurred

- Initiate actions to eliminate potential ignition sources

- Initiate actions to promote quick dilution of the concentrated gas to levels below the LFL.

\section{Types of Combustible Gas Detectors}

There are several types of combustible gas detectors, the most common being catalytic bead and infrared. Using catalytic bead detectors typically lowers capital cost because fewer detectors are needed but are not recommended because they require more frequent calibration and have a shorter life before internal components must be replaced. Infrared detectors, which are used most widely within the industry, are available as either a pointtype monitor or an open-path design. Point-type detectors detect an LCL level at a given point of the ceiling, whereas open path units detect an overall presence of gas within the beam. Open path detectors can reduce capital and maintenance costs in large garages, but typically require the use of some additional point-type detectors. There is no universally best approach, and the design of the system should be performed by a registered/licensed engineer in collaboration with the system manufacturer. A typical point-type infrared detector head is displayed in Figure 28.

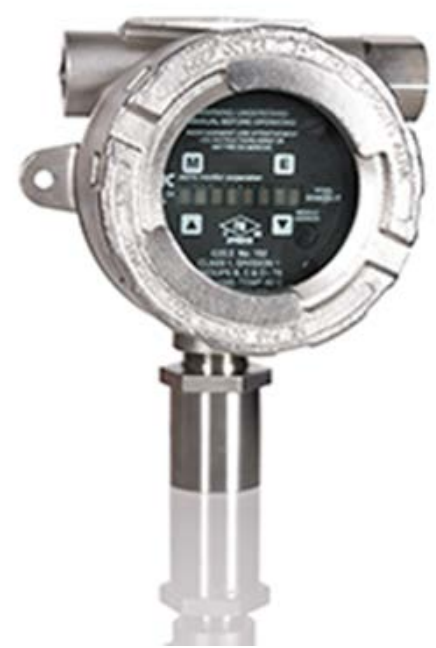

Figure 28. Typical infrared combustible gas detection head. Photo from Gladstein, Neandross \& Associates, NREL 44692 
The point-type infrared detector design is available from a number of manufacturers; a few are listed below:

- Det-tronics (Detector Electronics)

- Draeger

- Emerson

- General Monitors (Mine Safety Appliance [MSA])

- Honeywell

- Pemtech

- Safety Systems Technology

- Sensor Electronics

- Sierra Monitor Corporation

- Simark Controls.

Combustible gas detectors should be located near the highest point of the structure's ceiling. The number, location, and spacing of detectors must be determined by a registered/licensed design engineer and approved by the AHJ. Figure 29 displays a ceiling-mounted, combustible gas detection head.

Combustible gas detectors should be located near the highest point of the structure's ceiling. The number, location, and spacing of detectors must be determined by a registered/licensed design engineer and approved by the AHJ.

Because gas detectors are located on or near the building's ceiling, it is beneficial to select detectors that can be calibrated without requiring direct access to the unit. For

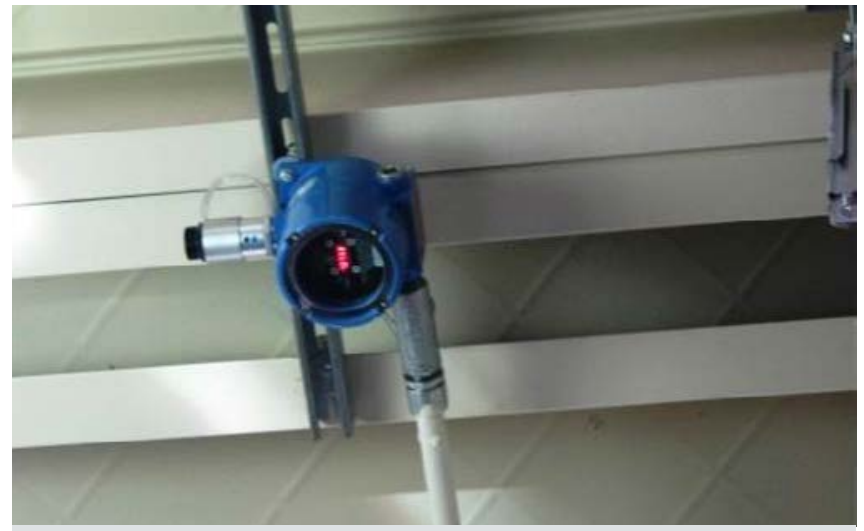

Figure 29. A ceiling-mounted combustible gas detection head. Photo from Gladstein, Neandross \& Associates, NREL 44683

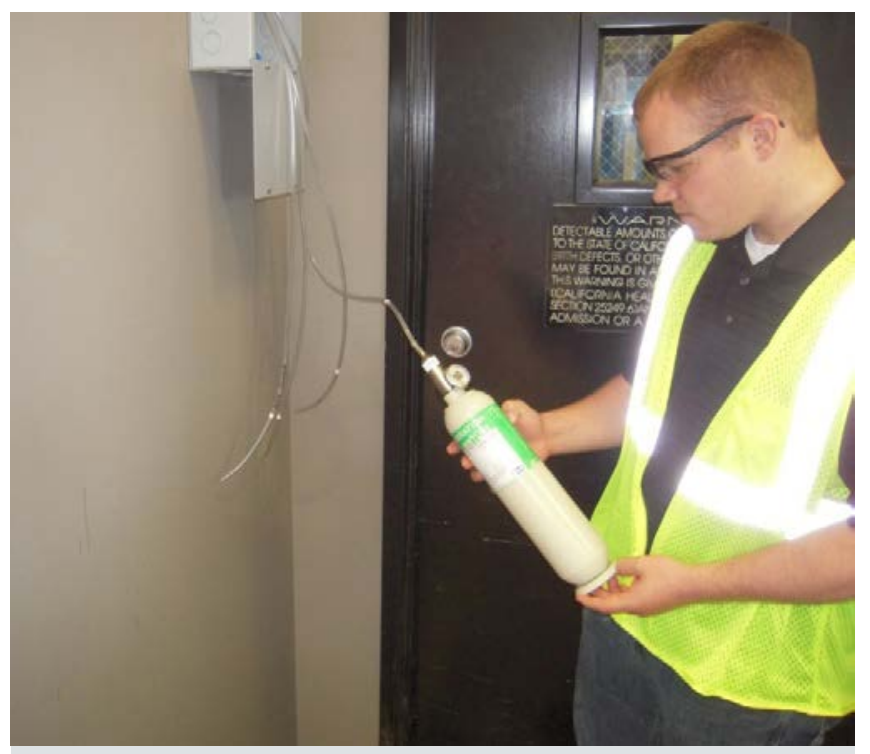

Figure 30. Gas detector calibration tubing. Photo from Gladstein, Neandross \& Associates, NREL 44684

example, the detectors can be calibrated by using tubing connected to the system at ground level to direct a calibration trace gas toward the unit (Figure 30).

The smell of the mercaptan in natural gas is a good indicator of a leak, but is only qualitative. Hand-held portable methane detectors are a quantitative method for identifying a leak. They are designed for localized gas detection and are a useful tool for technicians to have available when performing work in major vehicle repair garages because they provide a level of redundancy when working on the fuel system components. An example of a hand-held portable methane detector is shown in Figure 31. The readings of the detectors should be used with care, as they are prone to false positives. For this reason, they should be used in conjunction with other leak detection methods when work is being performed on vehicle fuel systems. Also, the smell of the mercaptan in natural gas is a good indicator of a leak, but is only qualitative.

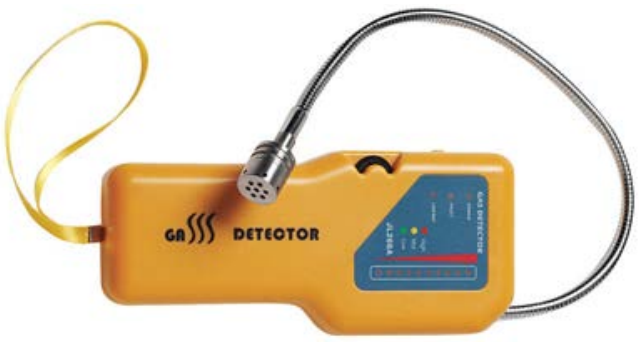

Figure 31. Typical hand-held portable methane detector. Photo from Gladstein, Neandross \& Associates, NREL 44691 


\section{Gas Detection and Alarm Systems}

Combustible gas detectors used in CNG vehicle maintenance facilities are designed and calibrated to measure methane concentrations as a percentage of the LFL $(5 \%$ for methane). Gas detectors read from $0 \%$ to $100 \%$ of the LFL, which means that a reading of $20 \%$ would indicate that the actual concentration of methane in the air is $1 \%$. Using this scale, methane detectors can be programmed to provide alarms at any concentration.

NFPA 30A states that a detection level of $25 \%$ of the LFL should be used to initiate alarms and other actions. The use of two gas detection levels rather than a single value has gained wide acceptance within the fire protection community and, with AHJ approval, should be incorporated into the operational design. By using two levels (i.e., $20 \%$ and $40 \%$ of the LFL), preliminary detection $(20 \%)$ occurs earlier so that protective actions can be initiated, followed by more extreme actions if concentrations reach the second level. A two-level approach is recommended because it greatly reduces the incidence of "nuisance" alarms while still ensuring personnel and facility safety.

If a two-level methane detection approach is used, the alarms connected to the detection system controls will activate first at the lower level, then again when the concentration reaches the higher level. It is typical for a beacon to be illuminated with a specific color indicating each level, and the alarm horn to be activated.

Alarm notification devices should be placed throughout the interior of the maintenance facility so that personnel can see them regardless of parked vehicles or other equipment inside. In addition, a similar alarm beacon and sound panel should be located outside the building and in the office. Some AHJs and facility managers also require alarm beacon and sound panels to be located within adjacent offices and administrative areas.
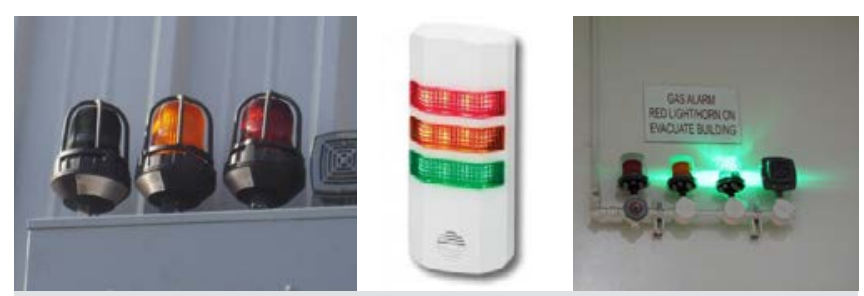

Figure 32. Examples of alarm status lights and horn panels (external and internal). Photo from Gladstein, Neandross \& Associates, NREL 44685, 44686, and 44692
Common alarm status lights and horn panels are shown in Figure 32.

It is recommended that the detection system be kept independent from the facility's fire detection and alarm systems. In addition, it is recommended that the two systems have distinctly different colored lights and alarm sounds.

\section{Control Systems}

Combustible gas detectors merely read the methane concentration in the air. Because of this, a control system is required for translating and interpreting the readings. As described previously, the three primary functions of the control system are to alert personnel of the existing conditions, increase ventilation within the structure, and eliminate possible ignition sources.

Alerting personnel is accomplished by means of the alarm beacons and sound horns. The control system can be programmed to activate the ventilation fans, open facility doors, and/or activate any other type of secondary ventilation equipment to increase air flow. The control system eliminates potential ignition sources by turning off (shunt tripping) the potentially hazardous electrical circuits and heating appliances within the facility.

As part of the retrofit process, selected electrical circuits should be modified so that isolation contactors or shunt trips are provided on all non-critical electrical circuitry. If shunt trips are used, it is recommended that they be activated only if the system detects a gas concentration of $40 \%$ LFL rather than the early-warning $20 \% \mathrm{LFL}$ as shunt trips require individual manual reset. If isolation contactors are used, isolation can occur at either 20\% LFL or $40 \%$ LFL as the system can automatically reset the circuits when the alarm clears. All electric circuits within the facility should be fitted with shunt trips or isolation contactors except the following:

- Electrical controls to the overhead doors that are selected to automatically open for the purpose of providing makeup air upon activation of the gas detection system

- Ventilation fans

- Gas detection and alarm systems and controls

- Emergency lighting

- Critical data collection or storage functions (computers, servers, etc.). 
Table 1. Possible Series of Actions Based on the Two Suggested Levels of Detection

\begin{tabular}{|c|c|c|c|}
\hline \multirow[b]{2}{*}{ Condition } & \multicolumn{3}{|c|}{ Methane Gas Concentration Level } \\
\hline & Normal & $20 \%$ LFL & $40 \%$ LFL \\
\hline Operation Lights - Green & On & Off & Off \\
\hline Operation Lights - Amber & Off & On & Off \\
\hline Operation Lights - Red & Off & Off & On \\
\hline Warning Horns & Off & On - Level 1 & On - Level 2 \\
\hline Strobe Alarms & On & On - Level 1 & On - Level 2 \\
\hline Supervisory Advisory & No & Yes & Yes \\
\hline Fire Department Callout & No & No & Yes \\
\hline Ventilation Fans & Manual & On & On \\
\hline Roll-Up Doors/Louvers & Manual & Open & Open \\
\hline Automatic Gas Valve & Open & Open & Closed \\
\hline Shunt Trip Building Loads & No & No & Yes \\
\hline $\begin{array}{l}\text { Automatic Reset of System if Fault } \\
\text { Clears }\end{array}$ & N/A & Yes & $\begin{array}{l}\text { No - Manual Reset } \\
\text { Required }\end{array}$ \\
\hline
\end{tabular}

Table 1 summarizes possible actions that may take place based on the two suggested levels of detection.

A status panel should be located outside of the building to allow emergency response teams to ascertain the internal conditions without having to enter the structure.

A master gas detection system control panel that displays system and detector status, historical information, and other variables should be located in the maintenance supervisor's office or other selected location. This programmable device is essentially the "brains" of the gas detection system. In addition, it is recommended that a status panel be located outside of the building. This will allow emergency response teams to ascertain the internal conditions without having to enter the structure. A typical gas detection system control panel is shown in Figure 33.
In the unlikely event that the gas detection system fails, the codes and regulations require that similar actions be initiated that would take place in the event of a release. These actions include activating the ventilation system (fans and makeup air), deactivating the heating systems, closing the gas valve, and sounding and lighting the alarms. These functions can be performed by accessing the gas detection system controller.

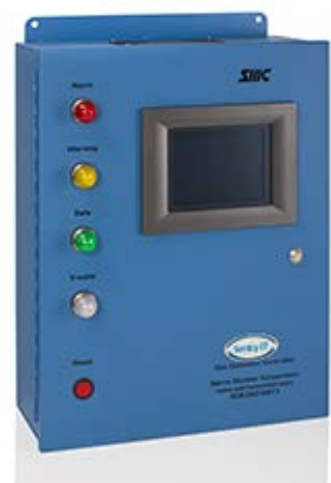

Figure 33. A combustible-gas detection system control panel. Photo from Gladstein, Neandross \& Associates, NREL 44688 


\section{Back-Up Power}

Some AHJs and/or insurance writers may require that back-up power be available if the power fails. Back-up batteries are usually selected to provide power to the gas detection and alarm system. However, batteries lack the power and duration needed to run other critical pieces of equipment, such as exhaust fans, make-up air, etc. To ensure there is sufficient power to operate all critical equipment, a stand-by generator equipped with an automatic transfer switch is typically employed. If the main power fails, the standby generator will automatically start and begin providing power to the facility. It is important to note that obtaining the necessary permits for an emergency generator may prove difficult in some regions due to air pollution concerns associated with engine exhaust. A typical emergency generator installation is shown in Figure 34.

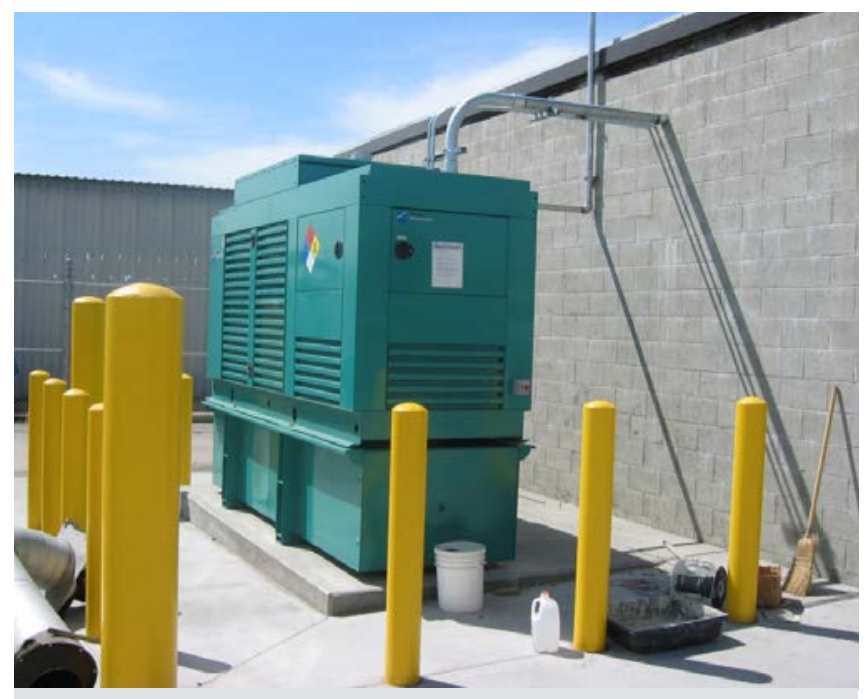

Figure 34. A typical emergency generator installation. Photo from Gladstein, Neandross \& Associates, NREL 44689
If the main power fails, it is unlikely that work will continue to be conducted within the facility. With all electricity (except the battery-powered detection and alarm system components) disconnected, it may be sufficient to merely evacuate the building and wait until the main power is restored. The gas detection system controls can be programmed to shunt-trip unnecessary equipment as a precaution. Therefore, if a leak occurs while the building is evacuated, potential ignition sources will be eliminated. The gas detection system is designed to alert personnel and prevent anyone from entering the maintenance facility until main power is restored.

It is essential that the gas detection, control, and alarm system be designed by an engineer registered/licensed within the state in which the facility is located. A registered/licensed engineer will be able to select equipment to ensure compatibility of components, evaluate operating conditions, and determine required functions. Approval must be obtained from the AHJ prior to preparing final designs and ordering equipment.

As noted above, the gas detection and control system is fully integrated with several functions within the facility, including shunt trips, activation of fans, opening of facility doors and other makeup air ventilation, and sounding of alarms. As an early step in the design process, a plot plan (layout) of the facility depicting the location of these functions and the hardware used to support them should be prepared for internal use and for preliminary approval by the AHJ. A typical layout showing these features is presented in Figure 35.

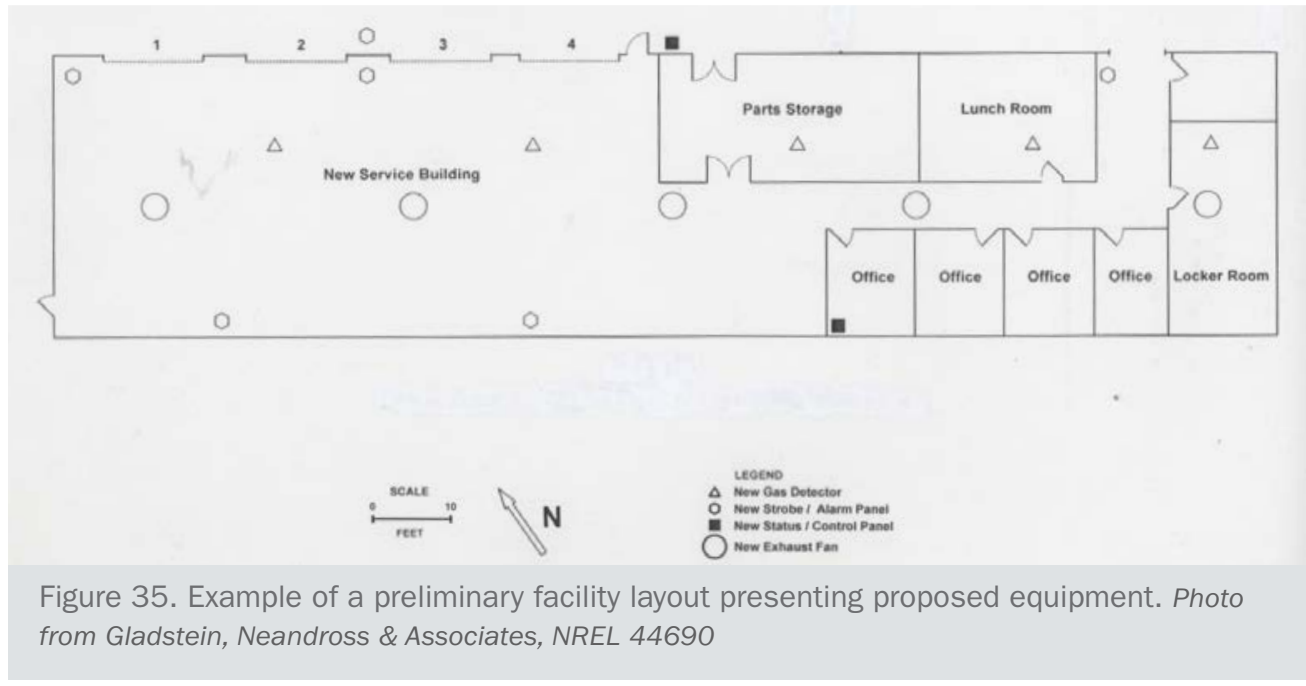




\section{Other Considerations}

In addition to vehicle maintenance facility modifications that must be implemented to comply with codes and regulations, there are a number of practical issues that should also be considered. Doing so early in the design process will make integration into the overall facility modification design easier.

\section{Vehicle Fuel System Defueling}

Removal of CNG from a vehicle's fuel tank (defueling) is a maintenance facility requirement that is often overlooked. Defueling is required whenever work on the CNG fuel tanks or non-isolated fuel system components is being performed within a major repair garage. Defueling is a practice that is performed infrequently but one that is required for certain types of repairs. For vehicles with fuel systems that allow one or more tanks to be completely isolated by means of manual shut-off valves, the remaining tanks that are not affected by the work being performed do not need to be defueled. Defueling a vehicle's fuel tank is not required if the fuel system is not involved in the repairs, no open flames are present, or welding is not performed. NFPA 52 and state fire codes offer guidance for the defueling operations.

Defueling is less complicated for maintenance facilities that are located on the same premises as a CNG fueling station because $\mathrm{CNG}$ fueling stations are often equipped with defueling capabilities. For maintenance facilities that are not colocated with a CNG fueling station, the vehicles must be taken offsite to be defueled prior to performing service or repairs and then fueled once the repairs have been made. This is often impractical because the vehicle would need to be towed into and out of the maintenance bay and refueled prior to after-service testing. Operations at some maintenance facilities use a portable, dolly-mounted CNG cylinder to slightly fill an empty CNG fuel tank. In this type of operation, the portable $\mathrm{CNG}$ cylinder provides a small amount of $\mathrm{CNG}$ to the vehicle and enables it to undergo post repair testing or to drive from the maintenance facility to the CNG fueling station.

Most commonly, defueling is performed using a "defueling" post or station. This device incorporates a hose and connections that allow the on-board tank to discharge through a vent to atmosphere or to a CNG station for facilities colocated with $\mathrm{CNG}$ fueling infrastructure. Defueling to a CNG station is preferred, as it enables the recapture of most of the defueled gas. Whenever possible, a vehicle's CNG tanks should be made as empty
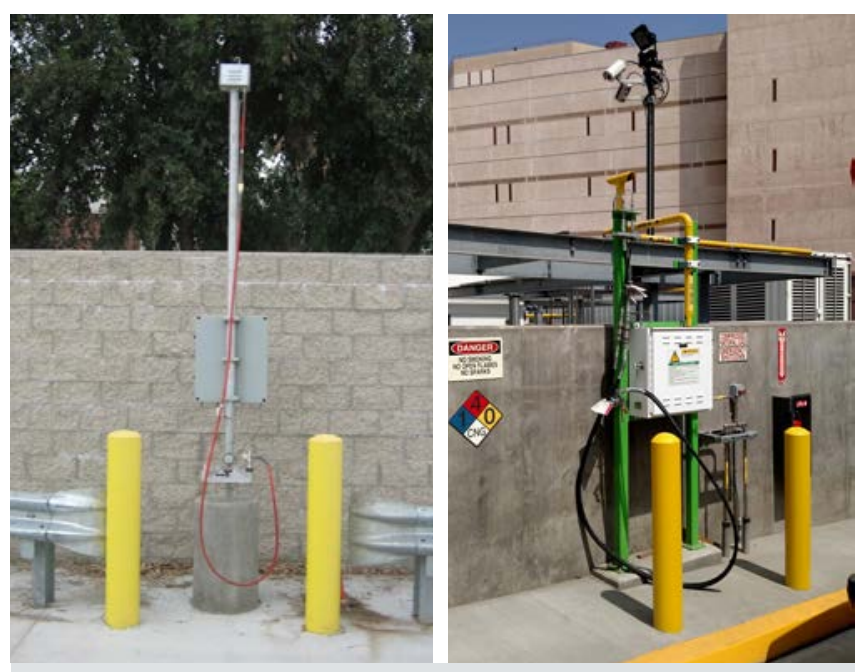

Figure 36 (left). A defueling post.

Figure 37 (right). A defueling station that defuels to a CNG station. Photos from Gladstein, Neandross \& Associates, NREL 44693 and 44694

as possible by consuming as much fuel as possible during operation or by transferring it to another vehicle prior to defueling the remaining $\mathrm{CNG}$ to the atmosphere. In addition, it is essential that the vehicle be connected to an earth ground before defueling operations begin. Typical defueling posts are displayed in Figure 36 and Figure 37. Portable equipment may also be available to defuel.

Another important defueling consideration pertains to the vehicle itself. The piping of a vehicle's fuel system may not be properly designed to allow for safe defueling. To ensure that vehicles can be safely defueled, fleets must inquire or specify that vehicles have this capability during the procurement process. Also note that there is more than one type of defueling connector, so this should be standardized across the fleet.

To ensure that vehicles can be safely defueled, ensure that vehicles have this capability during the procurement process.

\section{Engine Exhaust Extractors}

It is common practice for vehicle maintenance facilities to use engine exhaust extractors connected to a vehicle's exhaust whenever the engine is running. This ensures that a healthy and safe atmosphere is maintained within the facility. An example of an engine exhaust extractor is displayed in Figure 38. The exhaust from a natural gas engine is hotter than that from a diesel engine, and some exhaust extractor hoses are not compatible with the higher temperatures produced by natural gas engines. 


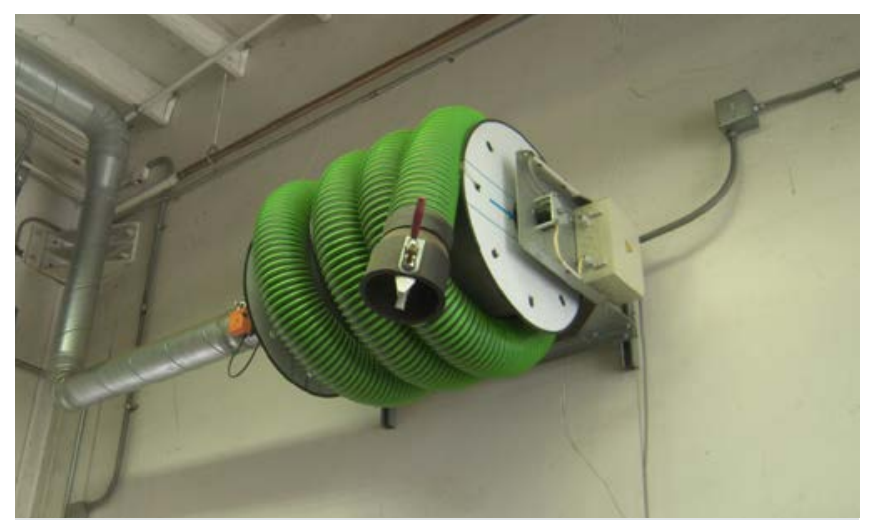

Figure 38. The exhaust from a natural gas engine is hotter than that from a diesel engine, so some exhaust extractor hoses may need to be replaced during the facility modifications. Photo from Gladstein, Neandross \& Associates, NREL 44695

As part of the facility modification process, the exhaust extractors should be evaluated to determine compatibility and prevent melting or igniting the lower-temperaturetolerant hose. Incompatible exhaust extractors should be replaced.

\section{Isolation of Non-Work Areas}

It is common for vehicle maintenance facilities to be designed with mezzanines or other non-work areas that are directly connected to areas where service and repairs are performed. These areas must be considered part of the facility modifications to protect them in the event of a natural gas release. This is typically achieved by installing barrier walls that can completely isolate the areas or by including the areas within the boundary of the gas detection and ventilation system. It may be more cost
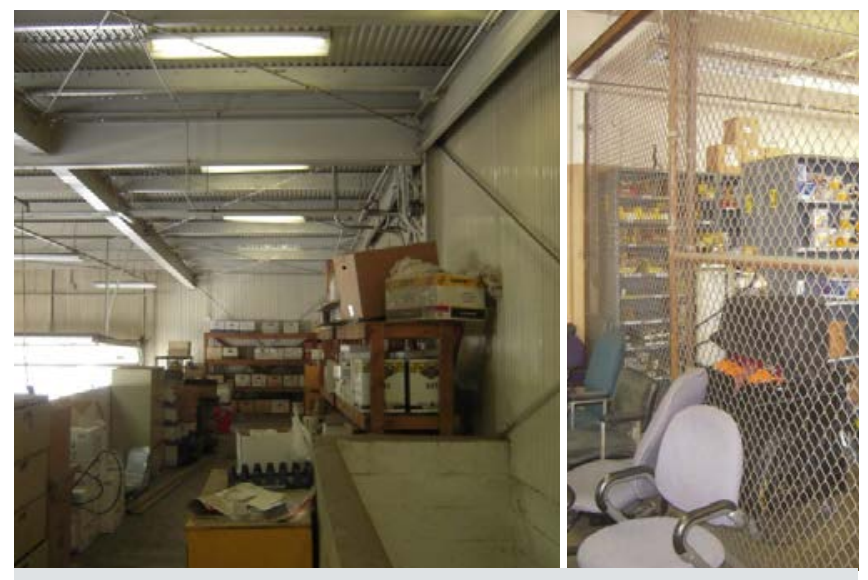

Figure 39. Typical mezzanine and adjacent storage areas. Photo from Gladstein, Neandross \& Associates , NREL 44889 and 44890 effective to isolate these areas with fixed barrier walls rather than fit them with gas detection and ventilation equipment to make the ceiling area safe. When barrier walls are installed, there is no need to provide a Class I, Division 2 rating to the ceiling of the area. It is essential that these areas be serviced with self-closing doors and that protocols are in place to prevent them from being left propped open. An example of a mezzanine and adjacent storage area that could be subject to isolation or inclusion in the gas detection and ventilation system is shown in Figure 39.

Some companies have developed isolation systems designed to meet NFPA and International Building Code guidelines. These systems allow for the isolation of one or two bays inside of a larger vehicle maintenance facility. Standard facility modifications to achieve compliance with the codes and regulations are performed only in the isolated bays. By isolating a smaller portion of the facility, modification costs are less than would be required for the larger area. Figure 40 presents a stylized representation of an isolation system installed in the corner of a larger vehicle maintenance facility. This approach could provide a low cost initial upgrade to service a limited fleet - the main garage upgrade could follow as the fleet grows.

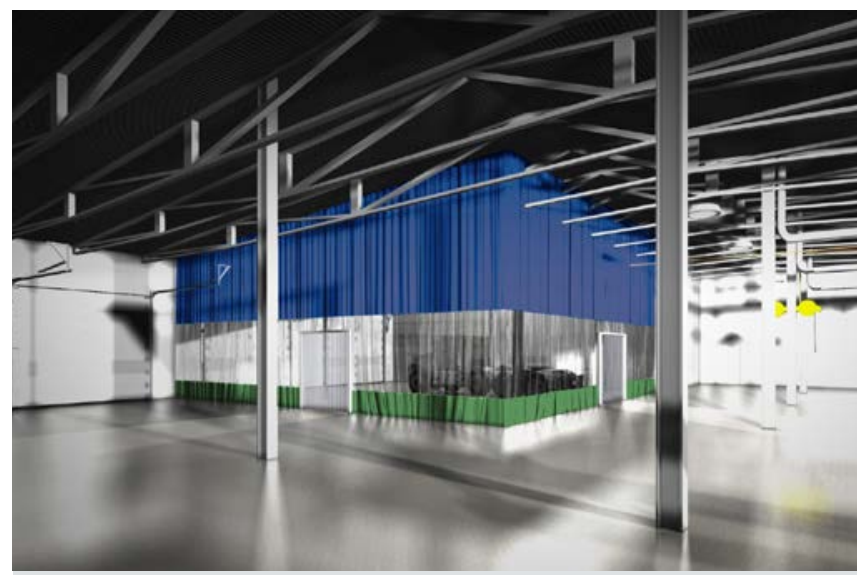

Figure 40. Representation of an isolation system. Photo from Gladstein, Neandross \& Associates, NREL 44891

\section{Automatic Shut-Off of Building Gas Supply}

If gas-fired space heaters or other appliances fueled by natural gas (e.g., steam cleaners, parts washing machines, water heaters) are used in the structure, it is recommended that an electrically controlled main gas valve be installed. This valve should be integrated with the gas detection system so that gas flow to the structure is automatically shut off when the alarm system is activated. Shutting down these appliances further reduces the 
number of potential sources of ignition in the facility in the event of a gas release. A typical electrically controlled gas shut-off valve is shown in Figure 41.

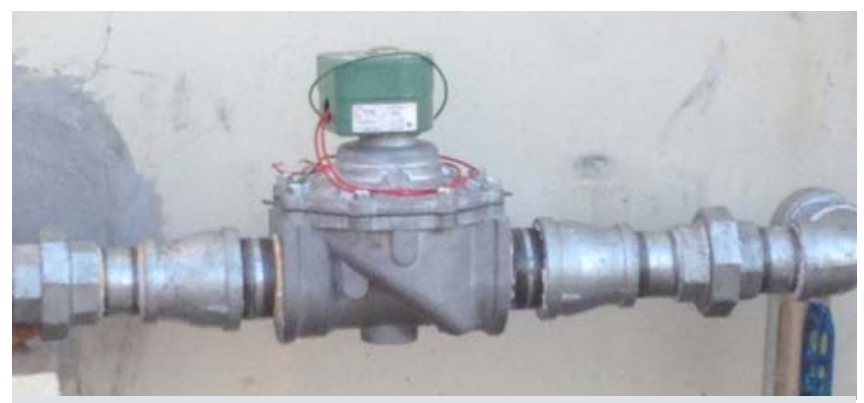

Figure 41. Electrically controlled main gas shut-off valve. Photo from Gladstein, Neandross \& Associates, NREL 44892

\section{Associated Bays}

Many maintenance facilities incorporate a vehicle wash bay(s) as part of the overall structure. Similarly, some maintenance facilities include a separate tire area where tires are stored and installed on vehicles. If these areas are isolated from the area where $\mathrm{CNG}$ vehicle service and repairs are performed (see Section 4.1, Paths of Migration) and access is controlled through self-closing doors, the areas would not be subject to compliance with the codes and regulations as long as vehicle maintenance activities are not performed in the bays.

Lubrication pits located in the vehicle maintenance facility are not required to have special protection in a $\mathrm{CNG}$ vehicle maintenance facility. Typically, these are ventilated as part of the codes and regulations for servicing conventional fuel vehicles. However, if the lubrication pits will be used to service LNG vehicles, they must be integrated with the gas detection and ventilation system design.

Paint bays are specifically included in the definition of a major repair garage. However, such bays may be used without CNG protection as long as painting is the only activity performed.

Paint bays are specifically included in the definition of a major repair garage. However, subject to approval by the AHJ, such bays may be used without CNG protection as long as painting is the only activity performed. If bodywork, hot work, or other similar activities are performed within the paint bay, the bay would not be exempt from
CNG protection. The registered/licensed engineer designing the facility upgrades should confirm that no baking of paint takes place in the paint bay as elevated temperatures can damage a vehicle's pressure relief device even if there is no gas in the tanks.

\section{Implementation}

This section is intended to assist the fleet or vehicle maintenance facility manager in determining the appropriate modifications for his/her specific location, as well as approaches to implementation.

\section{A competent, experienced vehicle maintenance} facility assessor should be engaged to perform the initial facility evaluation.

A competent, experienced vehicle maintenance facility assessor should be engaged to perform the initial evaluation of the facility. A number of firms employ CNG facility assessors. The local Clean Cities coordinator is also able to assist fleet managers with locating and selecting a qualified assessor. The assessor will work closely with fleet personnel, including service technicians, supervisors, and the fleet manager, to fully understand operating protocols and activities performed within the facility, including the work habits of operating personnel. In addition, the assessor will fully evaluate the electrical and mechanical systems within the facility to determine their compliance with codes and regulations and offer modification or replacement recommendations.

When the assessment is completed, the assessor will provide a report defining all of the modifications believed to be necessary for compliance with the codes and regulations based on the facility type (major or minor repair garage), existing equipment, and operating requirements of the facility. This initial report will form the basis for ongoing work.

An important part of the development of the final report will be early interaction with the AHJ. After the initial evaluation has been made, the preliminary conclusions should be communicated to the AHJ, preferably at a "site walk conference." Early involvement with the AHJ to obtain buy-in of the proposed modifications will generally ensure that all requirements have been captured in the final recommendations, which streamlines the permitting process. 
After the initial evaluation has been made, the preliminary conclusions should be communicated to the $\mathrm{AHJ}$. Early involvement with the AHJ will help ensure buyin and streamline the permitting process.

Typically, an assessor will not recommend a specific brand of equipment to be used but will instead provide performance specifications so that final designs can be made using the most cost-effective equipment option. The final report should provide an estimate for the work involved, including development of a request for proposal (RFP), design and permitting, equipment selection and procurement, installation, startup, and training.

Once the assessor's report is final and management approves the project, the first step is to prepare an RFP. This document will provide the basis for the design and construction contract(s) and all technical and administrative requirements for the project in a format that allows contractors to competitively bid for the work. The RFP is usually prepared by the assessor, but can also be prepared by the owner or a third party.

Two types of construction contracts are commonly used for this type of work. The first, known as a design-build (DB) contract, provides for final design, permitting, equipment procurement, and construction all to be performed by a single contractor. Under a DB contract, contractors propose a bid for the entire project. The second approach is a design-bid-build (DBB) contract. Under a DBB contract, design consultants will propose only the design services for the project. The completed design is then offered to construction contractors in a separate bidding process for the construction-related phases of the work.

Both approaches have advantages. DB contracting entails engaging only a single contractor, which can result in faster project implementation. DBB contracting tends to require more time because two contractors (the design contractor and the construction contractor) are typically engaged for their specific roles. One potential benefit of DBB is that it may provide the owner more control over the final scope and approach. DBB also ensures that the design team is looking out for the owner rather than for the contractor, which may be the case with the DB approach. The DBB approach also tends to allow more contractors to bid on the actual construction since they do not need to have design capabilities on their team. If it is a public project, selection of the DB or DBB alterna- tive may be dictated by policy. Private companies usually do not have this requirement.

Permit approval of the completed construction drawings is an essential part of the process. Typically, the construction contractor is responsible for obtaining building permits. When the AHJ is involved during the preliminary design process, the permitting usually goes smoothly and can be completed with minimal delays. In addition, obtaining buy-in from permitting authorities during the early stages of the project allows immediate purchase of long-lead equipment items, eliminating potential delays during the construction phase of the project.

During construction, the assessor is often involved in periodic monitoring of progress to ensure that the work is following the approved permit drawings and to act as a source of advice in the event of unforeseen problems. If change orders are required, the assessor can monitor them and act as a referee on behalf of the project owner. In either case, the project drawings should be reviewed by the assessor at various stages of design (i.e., $30 \%, 60 \%$, and $90 \%$ complete) to ensure compliance with the technical requirements of the RFP, sound engineering practices, appropriate equipment selection, and compliance with the appropriate codes and regulations.

The various systems are usually constructed and tested independently. Then, upon construction completion, systems integration and commissioning are performed to incorporate all of the components that interact.

Construction of the various systems - electrical, ventilation, gas detection, etc.--is usually completed independently, allowing each system to be tested and evaluated on a stand-alone basis. Upon construction completion, systems integration and commissioning are performed to incorporate all of the components that interact. The assessor is often involved in both commissioning and overall system startup. The vendor of the gas detection system should be involved in all phases of startup for this equipment, including calibration, troubleshooting, and testing.

The final step of the project is acceptance testing. Acceptance testing involves a thorough test program to evaluate the performance of the overall system, including a simulation of a gas detection event(s). Once initiated, the acceptance test evaluates all subsequent responses by the system controller to shunt trip selected loads, activate the ventilation and alarm systems, perform emergency 
The construction contractor is responsible for training personnel, including both practical training on system operations, and hands-on training to provide actual operation, troubleshooting, and maintenance experience.

callout, and other responses. This test must be witnessed by the AHJ as part of the permit sign-off process. Often the representative of the gas detection equipment manufacturer is also available during acceptance testing.

When all systems are "go," final as-built drawings will be prepared by the contractor and submitted to the project owner. The drawings are usually accompanied by all operating manuals, special tools, calibration gases, and other items that are required to maintain proper operation of the equipment. A full listing of all needed documentation should be noted as a requirement in the original project RFP.

Lastly, the construction contractor is responsible for training personnel, which should include both practical training to provide theoretical information about systems operations, and hands-on training to provide actual operation, troubleshooting, and maintenance experience on the systems and equipment.

\section{Project Costs and Schedules}

The cost and timing of $\mathrm{CNG}$ vehicle maintenance facility modifications vary substantially among projects because of differences in the facilities themselves. Modifications will be required to allow the servicing of $\mathrm{CNG}$ vehicles indoors, and in many cases additional work may be required by the $\mathrm{AHJ}$ - or desired by the facility operatorrelated to liquid fuel maintenance. It is common for previously postponed or deferred maintenance and capital improvements to be integrated into the project, becoming part of the overall facility modification. The age of a structure plays an important role in determining costs and schedule, as incumbent equipment or systems may not be amenable to modification and must sometimes be replaced. In some cases, it may be so costly to modify the existing facility that consideration should be given to erecting a new, compliant structure.

The completion timeline for maintenance facility modifications depends on the extent of the work required, budget allocation, permitting, and construction. Generally, equipment procurement is not a critical path item because most is readily available from a variety of vendors.
The following sections present an overview of the likely capital budgets and typical timelines required for implementing a facility modification project.

\section{Facility Modification Costs}

Facility modification costs depend on several factors, including:

- Number of bays/square footage to be modified

- Age and condition of the overall facility and the equipment within it

- Type of roof structure

- Associated attached or nearby structures

- Climate

- Inclusion of "non-protection" upgrades.

Facility modification costs depend on several factors:

- Number of bays or area to be modified

- Age and condition of the overall facility and the equipment within it

- Type of roof structure

- Associated attached or nearby structures

- Climate

- Inclusion of "non-protection” upgrades.

As noted previously, it is not necessary to modify every bay within a maintenance facility if not all bays will be used for CNG vehicle maintenance and repair. A careful evaluation of the operations to be conducted within the facility is needed to determine if it is possible to limit the number of bays treated. In some cases, isolating certain bays where $\mathrm{CNG}$ vehicle maintenance and repairs will be performed may be advisable to limit the costs. However, consideration should be given to the convenience of having all bays protected if the differential between the costs associated with isolating certain bays and modifying the entire facility is not substantial. Also, the incremental per-bay cost generally diminishes as the number of bays included in the project increases, thanks to common systems being used across all of the bays. Consequently, in some cases, it will be less costly to upgrade the entire facility rather than constructing partitions and isolating the HVAC systems. 
It is common for older facilities to be more costly to modify. Older facilities tend to need more equipment replaced because equipment and legacy modifications in an older facility are likely to be non-compliant with the $\mathrm{CNG}$ modifications required. It is usually more cost effective to completely replace older systems rather than modify them. In general, this applies to all systems, including the rating, location, and positioning of electrical systems and appliances; non-compliant heating systems; inadequate ventilation; structural concerns; and other considerations. It may be impractical to mount exhaust fans on the roof of an older building because of space or load-bearing limitations, requiring the use of more costly ground-mounted units fitted with ductwork. Similarly, the existing electrical supply and circuit panel may not have the load capacity to support the addition of new electrical appliances, particularly exhaust fans, requiring that the entire system be upgraded. The degree to which existing systems can be used as-is, with modifications, or require replacement is evaluated by the facility assessor.

Most CNG vehicle maintenance facilities include office and administrative areas, parts storage, wash bays, break rooms, and similar facilities. Others may be attached to a larger building that services all fleet operations. Although these auxiliary uses may not require protection, they will need to be suitably isolated from the maintenance and repair activities. In some cases, depending upon the requirements of the $\mathrm{AHJ}$ or insurance underwriters, they may need to be equipped with a gas detection system or alarm panels, increasing the cost of the project. The degree to which associated structures and work spaces need to be modified can be defined by the facility assessor and the AHJ.

The sophistication of the desired protection improvements and equipment will greatly affect project costs. If some of the options noted above are implemented to meet minimum code requirements, the total cost of the facility modification project will be minimized. For example, employing a simple detection system interlocked with ventilation and alarms will be less costly than a state-of-the-art control system using shunt tripping, an auxiliary generator, and other features. The degree of protection employed will be negotiated between the owner, the AHJ, and the facility insurance underwriter.

The relative cost difference between a cost-minimization approach and one that provides an increased degree of protection is generally low enough that the higher safety factor is well worth the incremental cost. Very sophisticated systems do not necessarily result in a greater degree of safety but may add flexibility to the operation and more control over the equipment employed.

Often, an approach that provides an increased degree of protection is well worth the incremental cost. Very sophisticated systems do not necessarily result in a greater degree of safety, but may add flexibility and control over the system and equipment.

It is common, when capital expenditures are required for CNG vehicle maintenance facility modifications, for building owners to want to include non-protection upgrades in the overall project. Such non-protection upgrades might include modernization of the offices and administrative areas, break rooms, or parts storage areas. Other upgrades may include the addition of skylights, new cranes, or similar improvements. These are often less expensive to include in the overall facility modification project than if they were stand-alone projects. Because of this, the merit of including them in the overall project needs to be considered when sufficient budgets are available.

Costs for facility modifications will generally include the following elements:

- Initial assessment

- Preliminary design and approval by the AHJ

- Preparation of a project RFP

- Selection of a DB or DBB contractor

- Detailed design of the facility

- Final permitting

- Equipment procurement including spare parts

- Modification of existing facilities and equipment

- Construction and new equipment installation

- Startup and system acceptance testing

- Training.

The cost for $\mathrm{CNG}$ vehicle maintenance facility modifications generally ranges from about $\$ 45,000$ to $\$ 100,000$ per bay. However, in rare cases, costs exceeding $\$ 200,000$ 
per bay have been reported. On average, the cost for modifying a four- to five-bay garage ranges from $\$ 75,000$ to $\$ 80,000$ per bay. As noted above, the incremental cost per bay diminishes as the number of bays included in the project increases due to common systems (gas detection controls, ventilation, heating, etc.) being used. Therefore, the average cost for modifying a 10-bay garage ranges from $\$ 60,000$ to $\$ 70,000$ per bay. Because modification costs are not inconsequential, it is imperative that the initial assessment and interaction with the AHJ be performed so that project budgets are established early in the process. There are many approaches to facility modifications, and these can be fully evaluated prior to committing to an approach.

It is also essential that upper management be apprised of the project and its costs so that the project can be included in the appropriate capital budgets.

\section{Facility Modification Timelines}

The primary driver of the overall readiness timeline for facility modifications is likely the date that indoor $\mathrm{CNG}$ vehicle maintenance and repairs will be required. Often, fleet operators will order CNG equipment without considering maintenance requirements and will find that vehicles have been delivered before the facility is ready. These vehicles must then be maintained out of doors or off the property. Typically, a maintenance facility can be modified within four to six months from the initial assessment. However, this largely depends on the sophistication of the systems requiring modification or replacement, permitting, and construction schedule. It has been reported that some facilities have taken as long as 24 months to complete. It is advisable to plan 12 to 24 months prior to the acquisition of $\mathrm{CNG}$ vehicles to ensure that the capital is in place to perform the modifications.

Typically, a maintenance facility can be modified within four to six months from the initial assessment. However, it is advisable to plan 12-24 months prior to the acquisition of CNG vehicles to ensure that the capital is in place to perform the modifications.

Considering the cost elements as presented above, Table 2 presents the average time for completing a facility modification project. 


\section{Table 2. CNG Vehicle Maintenance Facility Modification Schedule}

\section{Weeks}

Activity

$\begin{array}{lllllllllllllllllllllllllllllllllllllllllllll}1 & 2 & 3 & 4 & 5 & 6 & 7 & 8 & 9 & 1 & 1 & 1 & 1 & 1 & 1 & 1 & 1 & 1 & 1 & 2 & 2 & 2 & 2 & 2 & 2 & 2 & 2 & 2 & 2 & 3 & 3 & 3 & 3 & 3 & 3 & 3\end{array}$

Initial

Assessment

Preliminary

Design/AHJ

Approval

RFP Preparation

Contractor

Selection

Detailed Facility

Design

Final Permitting

Equipment

Procurement

Modification

Work

Construction

Startup/

Acceptance

Testing

Training

Appendix A: Specific Properties of Common Vehicle Fuels

\section{Table A1: Specific Properties of Common Vehicle Fuels}

\begin{tabular}{|c|c|c|c|c|c|}
\hline Compound & Formula & $\begin{array}{l}\text { Density }\left(\mathrm{lb} / \mathrm{ft}^{3}\right) \\
\text { Gases at STP }\end{array}$ & $\begin{array}{l}\text { Auto-Ignition } \\
\text { Temperature } \\
\left({ }^{\circ} \mathrm{F}\right)\end{array}$ & $\begin{array}{l}\text { Lower } \\
\text { Flammability Limit } \\
\text { (LFL) (\%) }\end{array}$ & $\begin{array}{l}\text { Upper } \\
\text { Flammability Limit } \\
\text { (UFL) (\%) }\end{array}$ \\
\hline CNG (Methane) & $\mathrm{CH}_{4}$ (majority) & 0.0447 & 1,004 & 5.3 & 15.0 \\
\hline Propane & $\mathrm{C}_{3} \mathrm{H}_{8}$ & 36.2 & $850-950$ & 2.2 & 9.5 \\
\hline Gasoline & $\mathrm{C}_{8} \mathrm{H}_{18}$ & 46.4 & 495 & 1.4 & 7.6 \\
\hline Diesel & - & 52.4 & 600 & 1.0 & 6.0 \\
\hline Hydrogen & $\mathrm{H}_{2}$ & 0.0056 & $1,050-1,080$ & 4.1 & 74.0 \\
\hline Air & - & 0.0806 & - & - & - \\
\hline
\end{tabular}




\section{Appendix B: Preliminary Maintenance Facility Assessment}

The following preliminary assessment tool is intended to help the maintenance facility manager determine if modifications are necessary based on anticipated fleet composition and operating protocols.

\section{Preliminary Assessment}

Do you plan to integrate CNG vehicles into your fleet?

When are the first CNG vehicles expected to be put in service?

Do you plan to convert a significant portion of your fleet to CNG?

Are you planning to outsource CNG vehicle maintenance and repairs or perform maintenance and repairs outside or under a canopy?

Will the maintenance and repairs be performed within your existing maintenance facility?

Will the maintenance facility be used for performing major repairs on CNG vehicles, such as engine overhauls, painting, body and fender work, or repairs that require emptying of the motor vehicle fuel tank or breaking open elements of the fuel system?

Repairs such as engine overhauls, painting, body and fender work, and other repairs that require emptying the motor vehicle fuel tank will result in the facility being classified as a major maintenance garage. To better understand the modifications required for a major maintenance garage, proceed to Appendix C, Minor/Major Maintenance Facility Assessment.

Will the maintenance facility be used only for performing minor repairs on CNG vehicles, such as lubrication, inspection, and minor automotive maintenance work, such as engine tune-ups, replacement of parts, fluid changes, brake system repairs, tire rotation, and similar routine maintenance work?

Repairs such as lubrication, inspection, and minor automotive maintenance work, such as engine tune-ups, replacement of parts, fluid changes, brake system repairs, tire rotation, and similar routine maintenance work result in the facility being classified as a minor maintenance garage. Classification as a minor maintenance garage may eliminate the need for some of the modifications required for a major garage. However, the specific modifications required will be largely determined by the AHJ because the codes and regulations do not explicitly state requirements for a minor maintenance garage. For this reason, proceed to Appendix C, Minor/Major Maintenance Facility Assessment. 


\section{Critical Decision Path of CNG Vehicle Maintenance Facility Modification}

Use answers from the preliminary assessment above to navigate the critical decision path in Figure B1. This will help determine whether facility modifications are required.

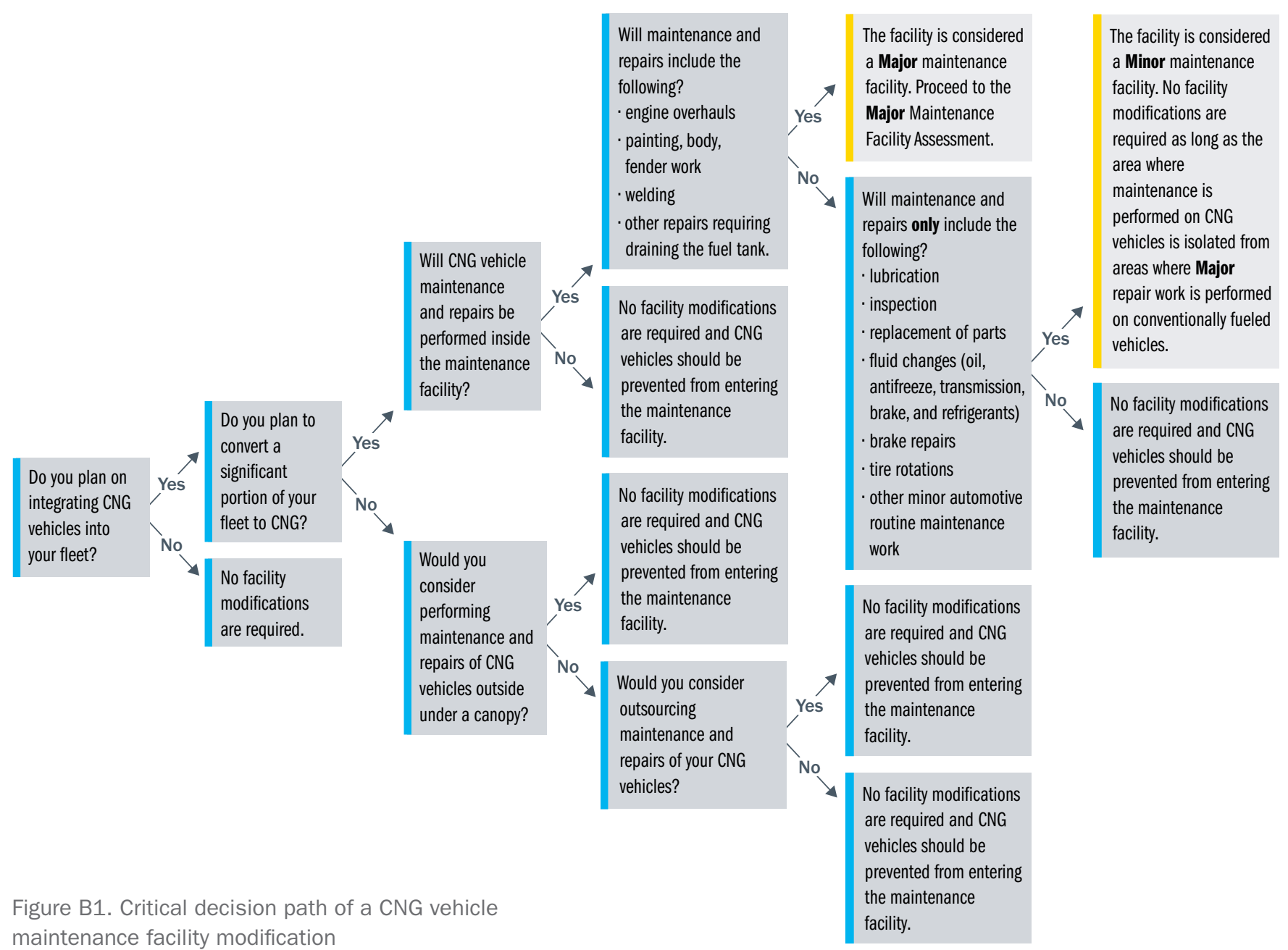




\section{Appendix C: Maintenance Facility Assessment}

The following assessment tool is intended to provide maintenance facility managers with an overall concept of which modifications will be required for their specific facility. As an initial step, the facility manager can use this tool as a guide to conduct an inspection of the facility and to initiate discussions with facility personnel.

This assessment tool has been developed according to the requirements for facilities at which major maintenance and repairs will take place. Existing codes and regulations do not specifically address the modifications required for facilities where minor maintenance and repairs will take place. Because of this, the specific modifications required for minor repair garages are often dependent on the authority having jurisdiction (AHJ).

\section{Overall Facility Assessment}

Will CNG maintenance and repairs be performed throughout the maintenance facility or only in certain bays or areas?

For fleets planning to convert a significant portion of their fleet to CNG, modifying the entire facility to perform maintenance and repairs on CNG vehicles will likely be most practical. However, fleets that are only converting a select portion of their fleet to CNG should consider the feasibility of isolating certain bays or areas of the facility for maintenance and repairs on CNG vehicles to minimize facility modification costs.

\section{Does the maintenance facility have any of the following characteristics?}

\section{- An open mezzanine or storage area}

- Doorways or open hallways to rooms or alcoves adjacent to the maintenance area

- Windows or openings to rooms or alcoves adjacent to the maintenance area.

Because the features above offer potential migration paths or accumulation areas, they will require attention during the facility modification process. There are two common ways of mitigating the hazards that the features present: they can be included with the facility modifications or isolated from the area where CNG vehicle maintenance and repairs are performed. Including them will require that they meet all the codes and regulations. However, they can be excluded from the facility modification process by adequate means of isolation. Common methods for isolating these features include installing barriers (walls, doors, etc.), pressurizing adjacent rooms, and sealing gaps or openings. Generally, isolation is more cost effective than including these features as part of the maintenance and repair area.

\section{Is it possible to isolate the area where major CNG vehicle maintenance will be performed from the rest of the facility?}

In some instances, a fleet may choose to only perform minor maintenance on CNG vehicles while continuing to perform major maintenance on conventionally fueled vehicles. In this case, the facility operator should assess the feasibility of isolating the area where CNG vehicle maintenance is performed from the area where major maintenance and repairs are performed on the conventionally fueled fleet. This form of isolation eliminates the need to modify the CNG vehicle maintenance area to comply with major standards. 


\section{Ventilation Assessment}

Does the maintenance facility have an existing ventilation system?

For facilities with an existing ventilation system, it is likely that modification of the ducting, fans, or operational protocols will be required. In the event that the facility does not have an existing ventilation system, a new system that complies with the codes and regulations must be installed as part of the facility modifications.

Does the ventilation system provide a continuous ventilation at the rate of 1 cubic foot per minute (cfm) for every 12 cubic feet $\left(\mathrm{ft}^{3}\right)$ of structural volume or a minimum of five air exchanges per hour?

The ventilation assessment should confirm the operational performance of the existing ventilation system. In the event that the existing system is not designed to provide continuous ventilation at the rate of $1 \mathrm{cfm}$ for every $12 \mathrm{ft}^{3}$ of structural volume or a minimum of five air exchanges per hour as required by NFPA 30A, the system will need to be upgraded as part of the modifications.

\section{For continuously operating ventilation systems, what type of control system is used?}

Modifications may be required depending on the existing control system for the continuously operating ventilation system. The system must be configured to be controlled by the combustible gas detection system or activated through the facility lighting system.

Does the maintenance facility have roof-mounted fans?

The fan blades and shrouds of roof-mounted fans must be constructed of non-sparking materials such as plastic or aluminum. Fan blades and shrouds not constructed of non-sparking materials will likely have to be replaced as part of the modification process.

Are the fan motors positioned so that the fan exhaust does not flow over the electric motor?

The type and location of the fan motors should be inspected and determined. Fan motors should not be located where the exhausting air flows over the fan motor unless the fan motor is classified as explosion-proof. Fans containing motors positioned in the path of exhausting air will have to be replaced with explosion-proof fan motors or fans that are configured so that exhausting air does not flow over the motor.

Are the exhaust fans or pick-up point(s) located at the highest point of the structure (either directly or through ducts)?

Exhaust fans and/or pick-up points must be positioned at the highest point of the structure to prevent natural gas from accumulating. If there are no exhaust fans or pick-up points affecting the highest point of the structure, additional fans and/or ducting must be included as part of the facility modifications.

Does the existing ventilation system provide a means for sufficient make-up air; e.g., in-wall louvers, sidewall fans, open doors, etc.?

A means for providing sufficient makeup air must be included in the facility modifications. 


\section{Space Heating Assessment}

Does the maintenance facility have an existing space heating system?

Facilities with an existing space heating system will need to have the system inspected to determine if modifications are required.

\section{Does the existing space heating system provide heat via forced air central heating?}

Forced air central heating systems present the least challenges for CNG vehicle maintenance facility compliance because they are not a potential ignition source. However, modifications may be required to accommodate changes to air flow rates if the ventilation system requires modification.

Does the existing space heating system provide heat via localized space heating (i.e., gas-fired infrared direct radiation or tube-type heaters, gas- (or propane-) fired devices equipped with fans, or electric infrared heating units)?

These types of localized space heating will present specific challenges for CNG vehicle maintenance facility compliance. Localized space heating is typically mounted to the walls of the facility or suspended from the ceiling, placing them in the path of rising natural gas. Many localized space heating apparatus contain an open flame, which is a direct ignition source. Even localized space heating apparatus that have contained flames pose a challenge because they commonly have exposed surfaces that can reach temperatures up to nearly $750^{\circ} \mathrm{F}$. Similarly, localized heating that relies on electric heating coils commonly has exposed surfaces that can reach temperatures up to nearly $750^{\circ} \mathrm{F}$. Localized space heating with any of these potential ignition sources will need to be replaced as part of the facility modifications. 
Does the maintenance facility have any standard-voltage conduit, junction boxes, or electrical fixtures within 18 " of the structure's ceiling?

Areas within 18" of the ceiling are designated as a Class I, Division 2 hazardous location. This designation will require that all electrical wiring, conduits, junction boxes, and electrical fixtures be replaced with explosion-proof equipment or relocated outside of the hazardous zone. Generally, it is less costly to relocate the equipment outside of the hazardous zone than it is to replace it with explosion-proof equipment. At the discretion of the $\mathrm{AHJ}$, conduits can be fitted with seal-offs rather than be relocated.

Does the maintenance facility have any low-voltage wiring conduits, junction boxes, or electrical appliances within 18" of the structure's ceiling; e.g., wiring for loudspeakers, security cameras, data transmission, alarms, etc.?

In some cases, the AHJ will not require that low-voltage wiring conduits, junction boxes, or electrical appliances be relocated to outside of the hazardous zone because these are non-sparking. However, these items must be approved by the AHJ before they can be excluded from the facility modifications.

Does the maintenance facility have lighting fixtures located within 18" of the structure's ceiling?

Lighting fixtures located within 18" of the structure's ceiling must be designated as explosion-proof, replaced with explosion proof lighting fixtures, or relocated to at least 18 " from the structure's ceiling.

Does the maintenance facility have other electrical fixtures located on walls or ceilings?

The electrical fixtures located on the walls may be in the path of migration for a rising release of natural gas. For this reason, the electrical fixtures must be relocated so that they are not in the path of migration.

Does the maintenance facility have electric door motors that will be activated during a gas detection event as a means of providing sufficient makeup air?

Electric door motors that are activated during a gas detection event must be designated as explosion-proof.

Does the maintenance facility have any electric door motors that will not be activated during a gas detection event?

Electric door motors that are not activated during a gas detection event are not required to be designated as explosion-proof. However, they must be shunt-tripped during a gas detection event to prevent a potential source of ignition.

Does the maintenance facility have overhead cranes or other items containing electric motors?

Because all electric motors (except those designated as explosion-proof) are a potential source of ignition, equipment containing electric motors such as overhead cranes must be shunt-tripped during a gas detection event. 


\section{Project Related Assessment}

\section{Are there other facility improvements currently being considered?}

Other facility improvements that are being considered should be evaluated for inclusion in the facility modification project. However, such other facility improvements should not be included in the project if they introduce potential project delays unrelated to the timeline for CNG vehicle maintenance modifications.

Has the cost of capital improvements for CNG vehicle facility modifications been appropriated in the budget?

It is essential that the capital costs of facility modification be included in the facility capital budget. Following the initial assessment of the likely modifications, the initial budgetary estimates and timelines should be revisited to confirm sufficient funding is available. Upper management must be "on board" with the proposed project.

\section{Have you located as-built drawings of the facility?}

As-built drawings will be essential for determining the specific modifications required. A full copy of these should be located so that they can be referred to throughout the project. 

Office of

ENERGY EFFICIENCY \&

RENEWABLE ENERGY

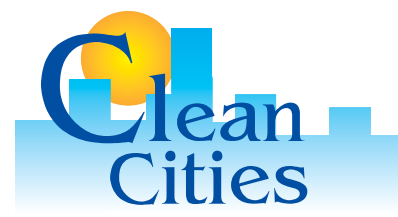

U.S. Department of Energy

For more information, visit:

cleancities.energy.gov

Clean Cities Technical Response Service 800-254-6735

technicalresponse@icfi.com

To view this and other Clean Cities publications online, visit cleancities.energy.gov/publications.

DOE/GO-102017-4918 · September 2017

Cover photos (clockwise from top), by Amy Glickson, NREL 45006; Amy Glickson, NREL 44909; Kendall Septon, NREL 44952 